\title{
ESSENTIAL NORMALITY - A UNIFIED APPROACH IN TERMS OF LOCAL DECOMPOSITIONS
}

\author{
YI WANG
}

\author{
Dedicated to the memory of Ronald G. Douglas
}

\begin{abstract}
In this paper, we define the asymptotic stable division property for submodules of $L_{a}^{2}\left(\mathbb{B}_{n}\right)$. We show that under a mild condition, a submodule with the asymptotic stable division property is $p$-essentially normal for all $p>n$. A new technique is developed to show that certain submodules have the asymptotic stable division property. This leads to a unified proof of most known results on essential normality of submodules as well as new results. In particular, we show that an ideal defines a $p$-essentially normal submodule of $L_{a}^{2}\left(\mathbb{B}_{n}\right), \forall p>n$, if its associated primary ideals are powers of prime ideals whose zero loci satisfy standard regularity conditions near the sphere.
\end{abstract}

\section{INTRODUCTION}

Let $\mathbb{B}_{n}$ be the open unit ball in $\mathbb{C}^{n}$. The Bergman space $L_{a}^{2}\left(\mathbb{B}_{n}\right)$ consists of all holomorphic functions $f$ on $\mathbb{B}_{n}$ such that

$$
\|f\|^{2}=\int_{\mathbb{B}_{n}}|f|^{2} d v<\infty
$$

Here $v$ denotes the normalized Lebesgue measure, i.e., $v\left(\mathbb{B}_{n}\right)=1$. For $i=1, \cdots, n$, the coordinate functions $z_{i}$ acts on $L_{a}^{2}\left(\mathbb{B}_{n}\right)$ by multiplication:

$$
M_{z_{i}} f=z_{i} f, \quad f \in L_{a}^{2}\left(\mathbb{B}_{n}\right) .
$$

The $n$-tuple of operators $\left(M_{z_{1}}, \cdots, M_{z_{n}}\right)$ are commuting and thus induces a Hilbert $\mathbb{C}\left[z_{1}, \cdots, z_{n}\right]$ module structure on $L_{a}^{2}\left(\mathbb{B}_{n}\right)$ :

$$
\mathbb{C}\left[z_{1}, \cdots, z_{n}\right] \times L_{a}^{2}\left(\mathbb{B}_{n}\right) \rightarrow L_{a}^{2}\left(\mathbb{B}_{n}\right), \quad(p, f) \mapsto p\left(M_{z_{1}}, \cdots, M_{z_{n}}\right) f=p f .
$$

For any $i, j=1, \cdots, n$, it is well known that the commutator $\left[M_{z_{i}}, M_{z_{j}}^{*}\right]$ belongs to the Schatten class $\mathcal{C}_{p}, \forall p>n$.

A closed subspace $\mathcal{P} \subset L_{a}^{2}\left(\mathbb{B}_{n}\right)$ that is invariant under $M_{z_{i}}, i=1, \cdots, n$, is called a (Hilbert) submodule of $L_{a}^{2}\left(\mathbb{B}_{n}\right)$. The commuting tuple $\left(R_{1}, \cdots, R_{n}\right)$, where $R_{i}=\left.M_{z_{i}}\right|_{\mathcal{P}}$, defines the module action on $\mathcal{P}$. Its orthogonal complement $\mathcal{Q}:=\mathcal{P}^{\perp}$ is called a quotient module of $L_{a}^{2}\left(\mathbb{B}_{n}\right)$. The module action on $\mathcal{Q}$ is defined by the tuple $\left(S_{1}, \cdots, S_{n}\right)$, where $S_{i}=\left.Q M_{z_{i}}\right|_{\mathcal{Q}}$. Here $Q$ denotes the projection operator onto $\mathcal{Q}$. For $p \geq 1$, we say $\mathcal{P}(\mathcal{Q})$ is $p$-essentially normal if $\left[R_{i}, R_{j}^{*}\right] \in \mathcal{C}_{p}\left(\left[S_{i}, S_{j}^{*}\right] \in \mathcal{C}_{p}\right)$.

For an ideal $I$ in $\mathbb{C}\left[z_{1}, \cdots, z_{n}\right]$, let $\mathcal{P}_{I}$ denote its closure in $L_{a}^{2}\left(\mathbb{B}_{n}\right)$. Then it is easy to see that $\mathcal{P}_{I}$ is a submodule of $L_{a}^{2}\left(\mathbb{B}_{n}\right)$. Therefore $\mathcal{Q}_{I}:=\mathcal{P}_{I}^{\perp}$ is a quotient module.

Arveson-Douglas Conjecture: Suppose $I$ is a homogeneous ideal in $\mathbb{C}\left[z_{1}, \cdots, z_{n}\right]$. Then the quotient module $\mathcal{Q}_{I}$ is $p$-essentially normal for all $p>\operatorname{dim}_{\mathbb{C}} Z(I)$.

Key words and phrases. essential normality, Arveson-Douglas Conjecture, Bergman space, asymptotic stable division. 
Remark 1.1. The Arveson-Douglas Conjecture was originally stated on the Drury-Arveson space $H_{n}^{2}$. Later it was shown that, for a homogeneous ideal I and any $p>n$, the $p$-essential normalities for the closures of I in the Drury-Arveson space, the Bergman space and the Hardy space, are equivalent. Closures of non-homogeneous ideals and non-polynomial generated submodules are also considered. Submodules on other domains were also considered.

In this paper, we consider submodules in the Bergman module. We will consider p-essential normality for $p>n$. For $p>n$, the p-essential normality of a submodule $\mathcal{P}$ is equivalent to the p-essential normality of its quotient module $\mathcal{Q}$.

The Arveson-Douglas Conjecture arises from Arveson's study of row contractions in multivariable operator theory [1]-[5]. Later, Douglas [7] showed that, given an essentially normal quotient module $\mathcal{Q}_{I}$, the short exact sequence

$$
0 \rightarrow \mathcal{K} \rightarrow C^{*}\left(\left\{S_{i}\right\}, I\right)+\mathcal{K} \rightarrow C\left(X_{I}\right) \rightarrow 0
$$

defines an element $\left[\mathcal{Q}_{I}\right]$ in the odd K-homology group $K_{1}\left(X_{I}\right)$ of a topological space $X_{I}$. One can show that $\overline{\mathbb{B}_{n} \cap Z(I)} \cap \partial \mathbb{B}_{n} \subset X_{I} \subset \partial \mathbb{B}_{n} \cap Z(I)$. In the case $I$ is homogeneous, $X_{I}=$ $Z(I) \cap \partial \mathbb{B}_{n}$. The element $\left[\mathcal{Q}_{I}\right]$ carries geometric information of $Z(I)$. This gives a new kind of index theorem. Moreover, a positive result of the Arveson-Douglas Conjecture will lead to an analytic Grothendieck-Riemann-Roch theorem allowing singularities [10].

The existing results of the Arveson-Douglas Conjecture can be roughly categorized into three types. The first type contains results concerning varieties of dimension 1 , or codimension 1 . In [15], Kuo and Wang proved the cases of homogeneous ideals $I$ when $n \leq 3$, or $\operatorname{dim} Z(I) \leq 1$, or $I$ is principal. Douglas and Wang $[8]$ showed that for a principal ideal $I$, not necessarily homogeneous, $\mathcal{P}_{I} \subset L_{a}^{2}\left(\mathbb{B}_{n}\right)$ is $p$-essentially normal for all $p>n$. Fang and Xia [13] 14] extended the results to polynomial-generated principal submodules of the Hardy space $H^{2}\left(\mathbb{B}_{n}\right)$, and, under additional assumptions, the Drury-Arveson space $H_{n}^{2}$. Douglas, Guo and the author 9] showed that a principal submodule of $L_{a}^{2}(\Omega)$ generated by a function $h \in \operatorname{Hol}(\bar{\Omega})$ is $p$-essentially normal for all $p>n$. Here $\Omega \subset \mathbb{C}^{n}$ is any bounded strongly pseudoconvex domain with smooth boundary. The result was extended to the Hardy spaces $H^{2}(\Omega)$ by the author and Xia [23].

The second type of results concern a geometric version of the Arveson-Douglas Conjecture. The results involve varieties with geometric conditions such as smoothness and transversallity on $\partial \mathbb{B}_{n}$. Engliš and Eschmeier [12] showed that, if a variety $V$ is homogeneous and its only possible singular point is the origin, then the radical ideal $I$ of all polynomials vanishing on $V$ defines a $p$-essentially normal quotient module for any $p>\operatorname{dim} V$. Douglas, Tang and $Y u$ showed that, if $I$ is radical and $Z(I)$ is a complete intersection space that is smooth on $\partial \mathbb{B}_{n}$, intersects transversely with $\partial \mathbb{B}_{n}$, then $\mathcal{Q}_{I}$ is essentially normal. Douglas and the author 11 showed that, if $I$ is a radical ideal and $Z(I)$ is smooth on $\partial \mathbb{B}_{n}$ and intersects transversely with $\partial \mathbb{B}_{n}$, then $\mathcal{Q}_{I}$ is $p$-essentially normal for all $p>2 \operatorname{dim} Z(I)$. The result was then refined to all $p>\operatorname{dim} Z(I)$ by the author and Xia [22].

The third type of results involve conditions that ensure decompositions of the submodules, or quotient modules into nice parts [17] 20] 21]. In particular, in [20], Shalit considered the stable division property of a submodule in $H_{n}^{2}$ and showed that a graded submodule with the stable division property is $p$-essentially normal for all $p>n$.

The aim of this paper is to provide a unified proof of most of the known Bergman-space results above. We define the asymptotic stable division property (Definition 3.1) and show that the asymptotic stable division property leads to essential normality. Our first main result is the following. 
Theorem 1.2 (Theorem $[3.2)$. Suppose $\mathcal{P}$ is a submodule of $L_{a}^{2}\left(\mathbb{B}_{n}\right)$ with the asymptotic stable division property. If the generating functions $h_{i}$ are all defined in a neighborhood of $\overline{\mathbb{B}_{n}}$, and the controlling constants $C_{i}, N_{i}$, determined by $h_{i}$ (as in Theorem [2.16), are uniformly bounded for all $i \in \Lambda$, then the submodule $\mathcal{P}$ is $p$-essentially normal for all $p>n$. In particular, if the generating functions $h_{i}$ are polynomials of uniformly bounded degrees, then $\mathcal{P}$ is p-essentially normal for all $p>n$.

The proof of Theorem 3.2 involves an inequality of a new type (Theorem 2.16) that first appeared in [9]. Since principal submodules and graded submodules with the stable division property have the asymptotic stable division property trivially, Theorem 3.2 provides a unified proof for the two types of results immediately.

We will also introduce technical hypotheses (Hypothesis 1) that lead to the asymptotic stable division property (Theorem 3.4). Then we will prove our second main result.

Theorem 1.3 (Theorem 4.1). Suppose I is an ideal in $\mathbb{C}\left[z_{1}, \cdots, z_{n}\right]$ with primary decomposition $I=\cap_{j=1}^{k} I_{j}^{m_{j}}$, where $I_{j}$ are prime ideals. Assume the following.

(1) For each $j=1, \cdots, k, Z\left(I_{j}\right)$ has no singular points on $\partial \mathbb{B}_{n}$ and intersects $\partial \mathbb{B}_{n}$ transversely.

(2) Any pair of the varieties $\left\{Z\left(I_{j}\right)\right\}$ does not intersect on $\partial \mathbb{B}_{n}$.

Then the submodule $\mathcal{P}_{I}$ has the asymptotic stable division property with generating elements $\left\{h_{i}\right\}$ being polynomials of uniformly bounded degrees. As a consequence, $\mathcal{P}_{I}$ is p-essentially normal for all $p>n$.

In [10, 5.2], the authors mentioned a plan of studying non-radical ideals. Theorem 1.3 partially accomplishes this goal, with a different approach.

Theorem 4.1 shows that, results of the second type also fit into this framework. The proof of Theorem 4.1 combines several techniques. First, we construct a covering that satisfies the bounded overlap condition for Bergman neighborhoods with large radius. The construction involves a radial-spherical decomposition method in 24. Then we construct a decomposition formula for each covering set. The generating functions are modified from local canonical defining functions of the variety. Combining these techniques, we show that the ideals in Theorem 4.1 satisfy Hypothesis 1, and therefore are $p$-essentially normal for all $p>n$.

In Section 2, we provide some tools that will be used in this paper. In Section 3 we introduce the asymptotic stable division property, and give a proof of Theorem [3.2. In Section 4 to Section 6, we prove Theorem 4.1. In the concluding remarks, we describe our future plans. In the Appendix, we prove some results involving algebraic sets. These results will be used mainly in Section 6.

Acknowledgment: The author would like to express her very great appreciation to Ronald G. Douglas in Texas A\& M University for the inspiring discussions and many support. She would also like to offer her special thanks to Jingbo Xia in SUNY Buffalo, for reading a draft of this paper carefully and providing many useful suggestions. The author would like to thank Guoliang Yu, Emil Straube and Zhizhang Xie in Texas A \& M University, Kunyu Guo in Fudan University, and Xiang Tang in Washington University in St. Louis, for the valuable suggestions and many supports. She would also like to thank Harold Boas, Gregory Pearlstein, J. M. Landsberg in Texas A\& M University, and Mohan Ramachandran in SUNY Buffalo, for answering many questions in several complex variables and algebraic geometry. 


\section{Preliminaries}

This section contains some basic tools that we are going to use in this paper. Besides the classic tools in the study of operators on the Bergman space, we will also use the theory of complex analytic sets substantively.

2.1. Arveson's Lemma. The following lemma provides an approach to the Arveson-Douglas Conjecture.

Lemma 2.1. [4] Suppose $\mathcal{P} \subseteq L_{a}^{2}\left(\mathbb{B}_{n}\right)$ is a submodule and $\mathcal{Q}$ is the corresponding quotient module. Then for any $p>n$, the following are equivalent.

(1) $\mathcal{P}$ is p-essentially normal;

(2) $\mathcal{Q}$ is p-essentially normal;

(3) $\left[M_{z_{i}}, P\right] \in \mathcal{C}_{2 p}, \forall i=1, \cdots, n$;

(4) $\left[M_{z_{i}}, Q\right] \in \mathcal{C}_{2 p}, \forall i=1, \cdots, n$.

Here $P, Q$ are the projections onto $\mathcal{P}$ and $\mathcal{Q}$, respectively.

Notice that

$$
\left[M_{z_{i}}, Q\right]^{*}=Q M_{z_{i}}^{*}-M_{z_{i}}^{*} Q=Q M_{z_{i}}^{*}-Q M_{z_{i}}^{*} Q=Q M_{z_{i}}^{*} P .
$$

Compared with the cross commutators $\left[M_{z_{i}}, M_{z_{j}}^{*}\right]$, the operators $Q M_{z_{i}}^{*} P$ are easier to work with. We will use Lemma 2.1 in the proofs in Section 3 ,

2.2. Complex Analytic Sets. The definitions and results come from [6].

Definition 2.2. Let $\Omega$ be a complex manifold. A set $A \subset \Omega$ is called a (complex) analytic subset of $\Omega$ if for each point $a \in \Omega$ there are a neighborhood $U$ of $a$ and functions $f_{1}, \cdots, f_{N}$ holomorphic in $U$ such that

$$
A \cap U=\left\{z \in U: f_{1}(z)=\cdots=f_{N}(z)=0\right\} .
$$

A point $a \in A$ is called regular if there is a neighborhood $V$ of $a$ in $\Omega$ such that $A \cap V$ is a complex submanifold of $\Omega$. The (complex) dimension $\operatorname{dim}_{a} A$ at $a$ is naturally defined to be the (complex) dimension of the manifold $A \cap V$.

A point $a \in A$ is called a singular point of $A$ if it is not regular. One can show that the set of regular points is dense in $A$. The dimension of $A$ at a singular point is defined as

$$
\operatorname{dim}_{a} A=\limsup _{\substack{z \rightarrow a \\ z \in \operatorname{reg} A}} \operatorname{dim}_{z} A .
$$

$A$ is said to be of pure dimension $p$ if $\operatorname{dim}_{a} A=p, \forall a \in A$.

In this paper, our main objects of study are algebraic sets in $\mathbb{C}^{n}$, i.e., zero loci of polynomials in $n$-variables. By Hilbert's Nullstellensatz, there is a one-to-one correspondence between algebraic sets and radical polynomial ideals, and irreducible algebraic sets correspond to prime ideals. We will also consider powers of radical ideals. Thus it is convenient to use the language of holomorphic chains.

Definition 2.3. A holomorphic chain on a complex manifold $\Omega$ is a formal, locally finite sum $\sum k_{j} A_{j}$, where $A_{j}$ are pairwise distinct irreducible analytic subsets in $\Omega$ and $k_{j} \neq 0$ are integers.

For $f \in \operatorname{Hol}(\Omega)$, we use the notation $\left.f\right|_{\sum k_{j} A_{j}}=0$ to indicate that $f$ is, locally, a linear combination of functions of the form $\Pi_{j} \Pi_{i=1}^{k_{j}} f_{i j}$, where $f_{i j}$ are holomorphic functions vanishing on $A_{j}$. 
Definition 2.4. A continuous map $f: X \rightarrow Y$ of topological spaces is called proper if the pre-image of every compact set $K \subseteq Y$ is a compact set in $X$. The spaces $X$ and $Y$ are assumed to be Hausdorff and locally compact.

Proper maps are important tools in the study of analytic sets. The following results will be used in the proofs.

Theorem 2.5. Let $A$ be an analytic set in $\mathbb{C}^{n}, a \in A, \operatorname{dim}_{a} A=p, 0<p<n, U$ a neighborhood of $a$, and $\pi: A \cap U \rightarrow U^{\prime} \subset \mathbb{C}^{p}, z \mapsto z^{\prime}:=\left(z_{1}, \cdots, z_{p}\right)$ a proper projection. Then there is an analytic subset $\sigma \subset U^{\prime}$ of dimension less than $p$ and a natural number $k$ such that

(1) $\pi: A \cap U \backslash \pi^{-1}(\sigma) \rightarrow U^{\prime} \backslash \sigma$ is a locally biholomorphic $k$-sheeted cover, in particular, $\# \pi^{-1}\left(z^{\prime}\right) \cap A \cap U=k$ for all $z^{\prime} \in U \backslash \sigma$.

(2) $\pi^{-1}(\sigma)$ is nowhere dense in $A_{(p)} \cap U$. Here $A_{(p)}=\left\{z \in A: \operatorname{dim}_{z} A=p\right\}$.

In particular, if $A$ is pure of dimension $p$, then $\pi^{-1}(\sigma)$ is nowhere dense in $A$. We say that $\pi$ defines a $k$-sheeted analytic cover. The set $\sigma$ is called the critical set of $\pi$.

A proper projection on a complex analytic set $A$ gives rise to a set of canonical defining functions [6].

Definition 2.6. (1) Let $a_{1}, \cdots, a_{k} \subset \mathbb{C}^{m}$, not necessarily distinct. We compose the polynomial

$$
P(z, w)=\left\langle z-a_{1}, w\right\rangle \cdots\left\langle z-a_{n}, w\right\rangle
$$

in the variable $(z, w) \in \mathbb{C}^{2 m}$. One can show that $P(z, w) \equiv{ }^{w} 0$ if and only if $z$ is one of the points $a_{1}, \cdots, a_{k}$. Suppose

$$
P(z, w)=\sum_{|\alpha|=k} P_{\alpha}(z) \bar{w}^{\alpha}
$$

where $\alpha$ denotes a multi-index $\left(\alpha_{1}, \cdots, \alpha_{m}\right)$. Then the condition $P(z, w) \equiv^{w} 0$ is equivalent to that $P_{\alpha}(z)=0, \forall \alpha,|\alpha|=k$. The polynomials $P_{\alpha}(z)$ are called the canonical defining functions for the system $a=\left\{a_{1}, \cdots, a_{k}\right\}$.

(2) More generally, suppose $A$ is an analytic subset of $U=U^{\prime} \times U^{\prime \prime} \subset \mathbb{C}^{n}$, where $U^{\prime} \subset \mathbb{C}^{p}$, $U^{\prime \prime} \subset \mathbb{C}^{m}$ and let $\pi:\left(z^{\prime}, z^{\prime \prime}\right) \mapsto z^{\prime} \in \mathbb{C}^{p}$. Suppose $\left.\pi\right|_{A}: A \rightarrow U^{\prime}$ is a $k$-sheeted analytic cover. Let $\sigma \subset \mathbb{C}^{p}$ be the critical set of $\left.\pi\right|_{A}$. For each $z^{\prime} \in U^{\prime} \backslash \sigma$,

$$
\pi^{-1}\left(z^{\prime}\right) \cap A \cap U=\left\{\left(z^{\prime}, a_{1}\left(z^{\prime}\right)\right), \cdots,\left(z^{\prime}, a_{k}\left(z^{\prime}\right)\right)\right\}
$$

where $a_{i}\left(z^{\prime}\right)$ are holomorphic functions defined in a small neighborhood of $z^{\prime}$. Define

$$
P_{\left.\pi\right|_{A}}(z, w)=\left\langle z^{\prime \prime}-a_{1}\left(z^{\prime}\right), w\right\rangle \cdots\left\langle z^{\prime \prime}-a_{k}\left(z^{\prime}\right), w\right\rangle, z \in U \backslash \pi^{-1}(\sigma), w \in \mathbb{C}^{m}
$$

and

$$
P_{\left.\pi\right|_{A}}(z, w)=\sum_{|\alpha|=k} P_{\left.\pi\right|_{A}, \alpha}(z) \overline{w^{\alpha}} .
$$

Here we write $\alpha=\left(\alpha_{p+1}, \cdots, \alpha_{n}\right)$ to be consistant with the coordinates in $\mathbb{C}^{n}$. The coefficients of powers of $z^{\prime \prime}$ in the functions $P_{\left.\pi\right|_{A}, \alpha}$ are locally bounded holomorphic functions on $U^{\prime} \backslash \sigma$. Since $\operatorname{dim} \sigma<p$, they can be uniquely extended to holomorphic functions on $U^{\prime}$. Therefore $P_{\left.\pi\right|_{A}, \alpha}$ extend to holomorphic functions on $U$ (in fact, on $U^{\prime} \times \mathbb{C}^{m}$ ). They are called the canonical defining functions for the projection $\pi$.

(3) One can also define canonical defining functions for holomorphic chains. We will only use the canonical defining functions for $m A$, where $m$ is a positive integer. Then we set 
$P_{\left.\pi\right|_{m A}}(z, w)=P_{\pi}(z, w)^{m}$ and $P_{\left.\pi\right|_{m A}}(z, w)=\sum_{|\alpha|=m k} P_{\left.\pi\right|_{m A}, \alpha}(z) \overline{w^{\alpha}}$. The functions $P_{\left.\pi\right|_{m A}, \alpha}(z)$ will be the canonical defining functions for the holomorphic chain $m A$.

Remark 2.7. We remark that in Definition [2.6, the functions $P_{\pi_{A}}$ and $P_{\left.\pi\right|_{A}, \alpha}$ are constructed under a specific choice of basis. Our estimates in this paper involve change of basis. It is convenient to generalize Definition 2.6 to the following "coordinate-free" form. Suppose $E=\left\{e_{1}, \cdots, e_{n}\right\}$ is an orthonormal basis of $\mathbb{C}^{n}$ and $\pi$ is the orthonormal projection onto $\operatorname{span}\left\{e_{1}, \cdots, e_{p}\right\}$. Suppose $\left.\pi\right|_{A}$ is proper. Define

$$
P_{\left.\pi\right|_{A}, E}(z, w)=\Pi_{a \in \pi^{-1} \pi(z) \cap A}\left\langle z-a, \sum_{i=p+1}^{n} w_{i} e_{i}\right\rangle, \quad z \in \mathbb{C}^{n}, w \in \mathbb{C}^{n-p} .
$$

Suppose $l$ is a unitary transformation on $\mathbb{C}^{n}$ and $\left.\pi\right|_{l(A)}$ is also proper. Let $E_{l}=\left\{l^{-1} e_{1}, \cdots, l^{-1} e_{n}\right\}$. Then

$P_{\left.\pi\right|_{l}(A), E}(l(z), w)=\Pi_{a \in \pi^{-1} \pi l(z) \cap l(A)}\left\langle l(z)-a, \sum_{i=p+1}^{n} w_{i} e_{i}\right\rangle=\Pi_{b \in l^{-1} \pi^{-1} \pi l(z) \cap A}\left\langle z-b, \sum_{i=p+1}^{n} w_{i} l^{-1}\left(e_{i}\right)\right\rangle$.

In other words,

$$
P_{\left.\pi\right|_{l}(A), E}(l(z), w)=P_{\left.l^{-1} \pi l\right|_{A}, E_{l}}(z, w) .
$$

We will use this fact in the proof of Lemma 6.1. In the subsequent discussions, we will omit the subscript $E$ where no confusion is caused.

2.3. Möbius Transform and Bergman Metric. For $z \in \mathbb{B}_{n}, z \neq 0$, let $P_{z}$ and $Q_{z}$ be the orthogonal projections from $\mathbb{C}^{n}$ to $\mathbb{C} z$ and $z^{\perp}$, respectively.

Definition 2.8. The Möbius transform $\varphi_{z}$ is defined by the formula

$$
\varphi_{z}(w)=\frac{z-P_{z}(w)-\left(1-|z|^{2}\right)^{1 / 2} Q_{z}(w)}{1-\langle w, z\rangle}, \quad w \in \mathbb{B}_{n}
$$

The following lemma contains some basic properties of the Möbius transform $\varphi_{z}$. One can find a proof in Chapter 2 of [19].

Lemma 2.9. If $a, z, w \in \mathbb{B}_{n}$, then

$$
1-\left\langle\varphi_{a}(z), \varphi_{a}(w)\right\rangle=\frac{(1-\langle a, a\rangle)(1-\langle z, w\rangle)}{(1-\langle z, a\rangle)(1-\langle a, w\rangle)}
$$

(2) As a consequence of (1),

$$
1-\left|\varphi_{a}(z)\right|^{2}=\frac{\left(1-|a|^{2}\right)\left(1-|z|^{2}\right)}{|1-\langle z, a\rangle|^{2}}
$$

(3) The Jacobian of the automorphism $\varphi_{z}$ is

$$
\left(J \varphi_{z}(w)\right)=\frac{\left(1-|z|^{2}\right)^{n+1}}{|1-\langle w, z\rangle|^{2(n+1)}} .
$$

Definition 2.10. The pseudo-hyperbolic metric $\rho$ is defined by

$$
\rho(z, w)=\left|\varphi_{z}(w)\right|, z, w \in \mathbb{B}_{n} .
$$


The hyperbolic metric $\beta$ is defined by

$$
\beta(z, w)=\frac{1}{2} \log \frac{1+\rho(z, w)}{1-\rho(z, w)}, z, w \in \mathbb{B}_{n} .
$$

$\beta$ is also called the Bergman metric on $\mathbb{B}_{n}$. For $r>0$ and $z \in \mathbb{B}_{n}$, denote

$$
D(z, r)=\{w: \beta(z, w)<r\} .
$$

The two metrics $\rho$ and $\beta$ define the same topology on $\mathbb{B}_{n}$. In the estimations, we will use whichever is more convenient. The following lemmas are straightforward to check. We omit the proofs.

Lemma 2.11. For $z, w \in \mathbb{B}_{n}$, we have

(1) $\beta(z, w) \in\left[-\frac{1}{2} \log \left(1-\rho^{2}(z, w)\right), \log 2-\frac{1}{2} \log \left(1-\rho^{2}(z, w)\right)\right)$.

(2) $1-\rho^{2}(z, w) \in\left[e^{-2 \beta(z, w)}, 4 e^{-2 \beta(z, w)}\right)$.

Lemma 2.12. For $z, w \in \mathbb{B}_{n}$, the following hold.

$$
|1-\langle z, w\rangle|>\frac{1}{2}\left(1-|z|^{2}\right)
$$

$$
1-\left|\varphi_{z}(w)\right|^{2} \in\left(\frac{1}{4}\left(1-|z|^{2}\right)\left(1-|w|^{2}\right), 4 \frac{1-|z|^{2}}{1-|w|^{2}}\right) .
$$

2.4. Spherical Distance. The following definitions and lemmas will be used in Section 5 ,

Definition 2.13. Let $S=\partial \mathbb{B}_{n}$ be the unit sphere in $\mathbb{C}^{n}$. For $\zeta, \xi \in S$, the spherical distance $d(\zeta, \xi)$ is defined by

$$
d(\zeta, \xi)=|1-\langle\zeta, \xi\rangle|^{1 / 2}
$$

Then $d$ defines a metric on $S$ (cf. [19]). For $\delta>0$, denote

$$
Q(\zeta, \delta)=\{\xi \in S: d(\xi, \zeta)<\delta\}
$$

Let $\sigma$ denote the normalized surface measure on $S$, i.e., $\sigma(S)=1$. For $z, w \in \mathbb{B}_{n}$, we will also write $d(z, w)=|1-\langle z, w\rangle|^{1 / 2}$. Then $d$ also satisfies the triangle inequality [19, Proposition $5.1 .2]$.

Lemma 2.14. [19, Proposition 5.1.4] When $n>1$, the ratio $\sigma\left(Q_{\delta}\right) / \delta^{2 n}$ increases from $2^{-n}$ to a finite limit $A_{0}$ as $\delta$ decreases from $\sqrt{2}$ to 0 .

On the punctured unit ball $\mathbb{B}_{n} \backslash\{0\}$, consider the projection

$$
\pi_{S}: \mathbb{B}_{n} \backslash\{0\} \rightarrow S, z \mapsto \frac{z}{|z|}
$$

For $z, w \in \mathbb{B}_{n}$, we will consider the spherical distance between their projections on $S$. Let us denote

$$
d_{S}(z, w)=d\left(\pi_{S}(z), \pi_{S}(w)\right)=\left|1-\left\langle\frac{z}{|z|}, \frac{w}{|w|}\right\rangle\right|^{1 / 2} .
$$

Lemma 2.15. For $z, w \in \mathbb{B}_{n}$, we have

(1) $d^{2}(z, w)<d_{S}^{2}(z, w)+\left(1-|z|^{2}\right)+\left(1-|w|^{2}\right)$.

(2) $d^{2}(z, w)>\frac{1}{2} d_{S}^{2}(z, w)$. 
Proof. By definition,

$$
d^{2}(z, w)=|1-\langle z, w\rangle|, d_{S}^{2}(z, w)=\left|1-\left\langle\frac{z}{|z|}, \frac{w}{|w|}\right\rangle\right| .
$$

Therefore,

$$
d^{2}(z, w) \leq(1-|z||w|)+|z||w|\left|1-\left\langle\frac{z}{|z|}, \frac{w}{|w|}\right\rangle\right|<\left(1-|z|^{2}\right)+\left(1-|w|^{2}\right)+d_{S}^{2}(z, w) .
$$

This proves (1).

The proof of (2) relies on the fact that $2|1-r c|>|1-c|$ for any $r \in(0,1)$ and $c \in \mathbb{C},|c|<1$. So

$$
d^{2}(z, w)=|1-\langle z, w\rangle|>\frac{1}{2}\left|1-\left\langle\frac{z}{|z|}, \frac{w}{|w|}\right\rangle\right|=\frac{1}{2} d_{S}^{2}(z, w) .
$$

This completes the proof.

2.5. An Inequality. In [9], the following theorem was proved, and then used to obtain $p$ essential normality of principal submodules.

Theorem 2.16. Suppose $h$ is a holomorphic function defined in a neighborhood of $\overline{\mathbb{B}_{n}}$. Then there exist a constant $C>0$ and a positive integer $N$, such that for any $z, w \in \mathbb{B}_{n}$ and any $f \in \operatorname{Hol}\left(\mathbb{B}_{n}\right)$, we have

$$
|h(z) f(w)| \leq C \frac{|1-\langle z, w\rangle|^{N}}{\left(1-|w|^{2}\right)^{n+1+N}} \int_{D(w, 1)}|h(\lambda) f(\lambda)| d v(\lambda) .
$$

The constants $C$ and $N$ depend on the function $h$. In the case when $h$ is a polynomial, the constants depend only on the degree of $h$. We provide a direct proof here.

Theorem 2.17. Suppose $p$ is a polynomial and $N=\operatorname{deg} p$. Then for any $f \in \operatorname{Hol}\left(\mathbb{B}_{n}\right)$ and $z, w \in \mathbb{B}_{n}$,

$$
|p(z) f(w)| \leq C \frac{|1-\langle z, w\rangle|^{N}}{\left(1-|w|^{2}\right)^{n+1+N}} \int_{D(w, 1)}|p(\lambda) f(\lambda)| d v(\lambda) .
$$

The constant $C$ depends only on $N$.

Proof. For $w \in \mathbb{B}_{n}, w \neq 0$ and $a, b>0$, denote

$$
Q_{w}(a, b)=\left\{z \in \mathbb{B}_{n}:\left|P_{w}(z)-w\right|<a\left(1-|w|^{2}\right),\left|Q_{w}(z)\right|<b\left(1-|w|^{2}\right)^{1 / 2}\right\} .
$$

From [19, 2.2.7], there exist $a, b$ such that $D(w, 1)$ contains $Q_{w}(a, b)$ for any $w \in \mathbb{B}_{n}, w \neq 0$.

For a polynomial $p$ with $\operatorname{deg} p=N$ and for $w \in \mathbb{B}_{n}$, choose an orthonormal basis $\left\{e_{1}, \cdots, e_{n}\right\}$ such that $e_{1}=\frac{w}{|w|}$. Then $w=(|w|, 0, \cdots, 0)$. In the case $w=0$, choose any orthonormal basis. For any multi-index $\alpha=\left(\alpha_{1}, \cdots, \alpha_{n}\right)$ such that $|\alpha| \leq N$, applying [8, Lemma 3.2] to the one variable polynomial $\partial^{\alpha_{2}} \cdots \partial^{\alpha_{n}} p\left(\cdot, w_{2}, \cdots, w_{n}\right)$, we get

$$
\begin{aligned}
& \left|\partial^{\alpha} p(|w|, 0, \cdots, 0) f(|w|, 0, \cdots, 0)\right| \\
\lesssim & \frac{1}{\left(1-|w|^{2}\right)^{\alpha_{1}+2}} \int_{\left|\lambda_{1}-\right| w||<a\left(1-|w|^{2}\right)}\left|\partial^{\alpha_{2}} \cdots \partial^{\alpha_{n}} p\left(\lambda_{1}, 0, \cdots, 0\right) f\left(\lambda_{1}, 0, \cdots, 0\right)\right| d v\left(\lambda_{1}\right) .
\end{aligned}
$$

Applying [8, Lemma 3.2] again to $\partial_{3}^{\alpha_{3}} \cdots \partial_{n}^{\alpha_{n}} p\left(\lambda_{1}, \cdot, w_{3}, \cdots, w_{n}\right)$, we get

$$
\begin{aligned}
& \left|\partial_{2}^{\alpha_{2}} \cdots \partial_{n}^{\alpha_{n}} p\left(\lambda_{1}, 0, \cdots, 0\right) f\left(\lambda_{1}, 0, \cdots, 0\right)\right| \\
\lesssim & \frac{1}{\left(1-|w|^{2}\right)^{\alpha_{2} / 2+1}} \int_{\left|\lambda_{2}\right|<\frac{b}{\sqrt{n-1}}\left(1-|w|^{2}\right)^{1 / 2}}\left|\partial_{3}^{\alpha_{3}} \cdots \partial_{n}^{\alpha_{n}} p\left(\lambda_{1}, \lambda_{2}, 0, \cdots, 0\right) f\left(\lambda_{1}, \lambda_{2}, 0, \cdots, 0\right)\right| d v\left(\lambda_{2}\right) .
\end{aligned}
$$


Inductively, for any $k=1, \cdots, n-1$,

$$
\begin{aligned}
& \left|\partial_{k+1}^{\alpha_{k+1}} \cdots \partial_{n}^{\alpha_{n}} p\left(\lambda_{1}, \cdots, \lambda_{k}, 0, \cdots, 0\right) f\left(\lambda_{1}, \cdots, \lambda_{k}, 0, \cdots, 0\right)\right| \\
\lesssim & \frac{1}{\left(1-|w|^{2}\right)^{\alpha_{k} / 2+1}} \int_{\left|\lambda_{k+1}\right|<\frac{b}{\sqrt{n-1}}\left(1-|w|^{2}\right)^{1 / 2}} \mid \partial_{k+2}^{\alpha_{k+2}} \cdots \partial_{n}^{\alpha_{n}} p\left(\lambda_{1}, \cdots, \lambda_{k+1}, 0, \cdots, 0\right) \\
& \quad f\left(\lambda_{1}, \cdots, \lambda_{k+1}, 0, \cdots, 0\right) \mid d v\left(\lambda_{k+1}\right) .
\end{aligned}
$$

Combining the inequalities above, we get

$$
\begin{aligned}
\left|\partial^{\alpha} p(w) f(w)\right| & \lesssim \frac{1}{\left(1-|w|^{2}\right)^{\alpha_{1}+\left|\alpha^{\prime}\right| / 2+n+1}} \int_{\substack{\left|\lambda_{1}-w_{1}\right|<a\left(1-|w|^{2}\right) \\
\left|\lambda^{\prime}\right|<b\left(1-|w|^{2}\right)^{1 / 2}}}|p(\lambda) f(\lambda)| d v(\lambda) \\
& \leq \frac{1}{\left(1-|w|^{2}\right)^{\alpha_{1}+\left|\alpha^{\prime}\right| / 2+n+1}} \int_{D(w, 1)}|p(\lambda) f(\lambda)| d v(\lambda) .
\end{aligned}
$$

Since $p(z)=\sum_{|\alpha| \leq N} c_{\alpha} \partial^{\alpha} p(w)(z-w)^{\alpha}$, where $c_{\alpha}$ are the Taylor coefficients, we have

$$
|p(z) f(w)| \lesssim \sum_{|\alpha| \leq N} \frac{\left|z_{1}-\right| w||^{\alpha_{1}}\left|z_{2}\right|^{\alpha_{2}} \cdots\left|z_{n}\right|^{\alpha_{n}}}{\left(1-|w|^{2}\right)^{\alpha_{1}+\left|\alpha^{\prime}\right| / 2+n+1}} \int_{D(w, 1)}|p(\lambda) f(\lambda)| d v(\lambda)
$$

Notice that

$$
\frac{\left|z_{1}-\right| w||^{2}}{|1-\langle z, w\rangle|^{2}}+\sum_{j=2}^{n}\left(1-|w|^{2}\right) \frac{\left|z_{j}\right|^{2}}{|1-\langle z, w\rangle|^{2}}=\left|\varphi_{w}(z)\right|^{2}<1
$$

We have

$$
\left|z_{1}-\right| w||<|1-\langle z, w\rangle|, \quad\left|z_{j}\right|<\frac{|1-\langle z, w\rangle|}{\left(1-|w|^{2}\right)^{1 / 2}}, j=2, \cdots, n .
$$

Therefore

$$
\begin{aligned}
|p(z) f(w)| & \lesssim \sum_{|\alpha| \leq N} \frac{|1-\langle z, w\rangle|^{\alpha_{1}+\left|\alpha^{\prime}\right| / 2}}{\left(1-|w|^{2}\right)^{\alpha_{1}+\left|\alpha^{\prime}\right| / 2+n+1}} \int_{D(w, 1)}|p(\lambda) f(\lambda)| d v(\lambda) \\
& \lesssim \frac{|1-\langle z, w\rangle|^{N}}{\left(1-|w|^{2}\right)^{N+n+1}} \int_{D(w, 1)}|p(\lambda) f(\lambda)| d v(\lambda) .
\end{aligned}
$$

From the previous argument, we know that the controlling constant depends only on $N$. This completes the proof.

2.6. Some Useful Computations. The following inequality will be useful in subsequent estimates. Its proof is a direct application of the Hölder's inequality.

Lemma 2.18. For a positive integer $M$ and $a_{1}, \cdots, a_{M}>0$,

$$
\left(a_{1}+a_{2}+\cdots+a_{M}\right)^{2} \leq M\left(a_{1}^{2}+a_{2}^{2}+\cdots+a_{M}^{2}\right) .
$$

We will use the following version of Schur's test.

Lemma 2.19. Let $(X, d \mu)$ and $(X, d \nu)$ be measure spaces and $T$ be an integral operator with non-negative integral kernel $K(x, y)$,

$$
T f(x)=\int_{X} f(y) K(x, y) d \mu(y), \quad x, y \in X .
$$


Suppose there exist a $\mu$-measurable positive function $h$ and a $\nu$-measurable positive function $g$ on $X$ such that

$$
\int_{X} h(y) K(x, y) d \mu(y) \leq A g(x), \quad \text { a.e. }[\nu],
$$

and

$$
\int_{X} g(x) K(x, y) d \nu(x) \leq B h(y), \quad \text { a.e. }[\mu] .
$$

then $T$ defines a bounded operator from $L^{2}(X, d \mu)$ to $L^{2}(X, d \nu)$ and $\|T\| \leq A^{1 / 2} B^{1 / 2}$.

We want to apply Schur's test to operators determined by the following integral kernels. For any $r>0$ and non-negative integers $l>0,0<d<n$, define

$$
\begin{gathered}
T_{l} f(z)=\int_{\mathbb{B}_{n}} f(w) \frac{\left(1-|w|^{2}\right)^{l}}{|1-\langle z, w\rangle|^{n+1+l}} d v(w), \\
T_{d, l} f(z)=\int_{\mathbb{B}_{n}} f(w) \frac{\left(1-\left|w^{\prime}\right|^{2}\right)^{l}}{\left|1-\left\langle z^{\prime}, w^{\prime}\right\rangle\right|^{n+1+l}} d v(w), \\
\tilde{T}_{l} f(z)=\int_{\mathbb{B}_{n}} f(w) \frac{\left(1-|w|^{2}\right)^{l}}{|1-\langle z, w\rangle|^{n+1 / 2+l}} d v(w), \\
T_{l}^{r} f(z)=\int_{D(z, r)} f(w) \frac{\left(1-|w|^{2}\right)^{l}}{(1-\langle z, w\rangle)^{n+1+l}} d v(w), \\
R_{l}^{r} f(z)=\int_{D(z, r)^{c}} f(w) \frac{\left(1-|w|^{2}\right)^{l}}{|1-\langle z, w\rangle|^{n+1+l}} d v(w) \\
R_{l, d}^{r} f(z)=\int_{D\left(\left(z^{\prime}, 0\right), r\right)^{c}} f(w) \frac{\left(1-\left|w^{\prime}\right|^{2}\right)^{l}}{\left|1-\left\langle z^{\prime}, w^{\prime}\right\rangle\right|^{n+1+l}} d v(w) .
\end{gathered}
$$

Here $w^{\prime}=\left(w_{1}, \cdots, w_{d}\right)$.

Lemma 2.20. For the operators defined above, the following hold.

(1) For any positive integer $l$ and $0<d<n, T_{l}, T_{d, l}$ define bounded operators on $L^{2}\left(\mathbb{B}_{n}\right)$. If $l \geq 1$, then $T_{l}$ also defines a bounded operator on $L^{2}\left(\mathbb{B}_{n},\left(1-|z|^{2}\right) d v(z)\right)$.

(2) For $\bar{l} \geq 0$, $\tilde{T}_{l}$ defines a bounded operator from $L^{2}\left(\mathbb{B}_{n},\left(1-|z|^{2}\right) d v(z)\right)$ to $L^{2}\left(\mathbb{B}_{n}\right)$.

(3) For any positive integer $l$ and for any $f \in \operatorname{Hol}\left(\mathbb{B}_{n}\right)$,

$$
T_{l}^{r} f(z)=c_{r, l} f(z),
$$

where $c_{r, l}=\int_{D(0, r)}\left(1-|z|^{2}\right)^{l} d v(z)$.

(4) For any $0<d<n$, any positive integer $l$ and any $r>0$, the operators $R_{l}^{r}$ and $R_{l, d}^{r}$ define bounded operators on $L_{a}^{2}\left(\mathbb{B}_{n}\right)$. Moreover,

$$
\max \left\{\left\|R_{l}^{r}\right\|,\left\|R_{l, d}^{r}\right\|\right\} \leq \epsilon_{r, l},
$$

where we have $\epsilon_{r, l} \rightarrow 0$ as $r \rightarrow \infty$, for fixed $l$.

Proof. We will only prove the statements for $T_{d, l}, \tilde{T}_{l}, T_{l}^{r}$ and $R_{l, d}^{r}$. We will use the Rudin-Forelli estimates [19, Proposition 1.4.10]. 
Let $h(z)=\left(1-\left|z^{\prime}\right|^{2}\right)^{-1 / 2}$. Then

$$
\begin{aligned}
& \int_{\mathbb{B}_{n}} \frac{\left(1-\left|w^{\prime}\right|^{2}\right)^{l}}{\left|1-\left\langle z^{\prime}, w^{\prime}\right\rangle\right|^{n+1+l}} h(w) d v(w) \\
= & \int_{w^{\prime} \in \mathbb{B}_{d}} \frac{\left(1-\left|w^{\prime}\right|^{2}\right)^{l-1 / 2}}{\left|1-\left\langle z^{\prime}, w^{\prime}\right\rangle\right|^{n+1+l}} \int_{\left|w^{\prime \prime}\right|^{2}<1-\left|w^{\prime}\right|^{2}} 1 d v_{n-d}\left(w^{\prime \prime}\right) d v_{d}\left(w^{\prime}\right) \\
\approx & \int_{\mathbb{B}_{d}} \frac{\left(1-\left|w^{\prime}\right|^{2}\right)^{l+n-d-1 / 2}}{\left|1-\left\langle z^{\prime}, w^{\prime}\right\rangle\right|^{n+1+l}} d v_{d}\left(w^{\prime}\right) \\
\lesssim & \left(1-\left|z^{\prime}\right|^{2}\right)^{-1 / 2}=h(z) .
\end{aligned}
$$

Here $v_{k}$ denotes the Lebesgue measure on $\mathbb{C}^{k}$. Similarly, we have

$$
\int_{\mathbb{B}_{n}} \frac{\left(1-\left|w^{\prime}\right|^{2}\right)^{l}}{\left|1-\left\langle z^{\prime}, w^{\prime}\right\rangle\right|^{n+1+l}} h(z) d v(z) \lesssim h(w) .
$$

This proves (1).

To prove $(2)$, take $h(z)=\left(1-|z|^{2}\right)^{-1 / 2}$ and $g(w)=\left(1-|w|^{2}\right)^{-1}$. We omit the calculations.

For any $f \in \operatorname{Hol}\left(\mathbb{B}_{n}\right)$ and any $z \in \mathbb{B}_{n}$,

$$
\begin{aligned}
T_{l}^{r} f(z) & =\int_{D(z, r)} f(w) \frac{\left(1-|w|^{2}\right)^{l}}{(1-\langle z, w\rangle)^{n+1+l}} d v(w) \\
& =\int_{D(0, r)} f \circ \varphi_{z}(\lambda) \frac{\left(1-\left|\varphi_{z}(\lambda)\right|^{2}\right)^{l}}{\left(1-\left\langle z, \varphi_{z}(\lambda)\right\rangle\right)^{n+1+l}} \frac{\left(1-|z|^{2}\right)^{n+1}}{|1-\langle z, \lambda\rangle|^{2(n+1)}} d v(\lambda) \\
& =\int_{D(0, r)} f \circ \varphi_{z}(\lambda) \frac{\left(1-|\lambda|^{2}\right)^{l}}{(1-\langle\lambda, z\rangle)^{n+1+l}} d v(\lambda) \\
& =c_{r, l} f(z) .
\end{aligned}
$$

This proves (3).

Let $E=\left\{(z, w): \beta\left(w,\left(z^{\prime}, 0\right)\right)>r\right\}$. Denote $\beta_{d}$ the Bergman metric on $\mathbb{B}_{d}$. If $(z, w) \in E$, then $1-\left|\varphi_{\left(z^{\prime}, 0\right)}(w)\right|^{2}<4 e^{-2 r}$. Since

$$
\begin{aligned}
1-\left|\varphi_{\left(z^{\prime}, 0\right)}(w)\right|^{2} & =\frac{\left(1-\left|z^{\prime}\right|^{2}\right)\left(1-|w|^{2}\right)}{\left|1-\left\langle z^{\prime}, w^{\prime}\right\rangle\right|^{2}} \\
& =\left(1-\left|\varphi_{z^{\prime}}\left(w^{\prime}\right)\right|^{2}\right) \frac{1-|w|^{2}}{1-\left|w^{\prime}\right|^{2}}
\end{aligned}
$$

either $1-\left|\varphi_{z^{\prime}}\left(w^{\prime}\right)\right|^{2}<2 e^{-r}$ or $\frac{1-|w|^{2}}{1-\left|w^{\prime}\right|^{2}}<2 e^{-r}$. Let $E_{1}=\left\{(z, w): \beta_{d}\left(z^{\prime}, w^{\prime}\right)>\frac{1}{2} r\right\}$ and $E_{2}=\left\{(z, w): \frac{1-|w|^{2}}{1-\left|w^{\prime}\right|^{2}}<2 e^{-r}\right\}$. Then $E \subset E_{1} \cup E_{2}$. It is easy to show that the integral kernels $\chi_{E_{1}} \frac{\left(1-\left|w^{\prime}\right|^{2}\right)^{l}}{\left|1-\left\langle z^{\prime}, w^{\prime}\right\rangle\right|^{n+1+l}}$ and $\chi_{E_{2}} \frac{\left(1-\left|w^{\prime}\right|^{2}\right)^{l}}{\left|1-\left\langle z^{\prime}, w^{\prime}\right\rangle\right|^{n+1+l}}$ define bounded operators with norms tending to 0 as $r \rightarrow \infty$. This proves (4).

We will also use the weighted Bergman norm. For $l$ a positive integer and $f \in \operatorname{Hol}\left(\mathbb{B}_{n}\right)$,

$$
\|f\|_{L_{a, l}^{2}}^{2}=\int_{\mathbb{B}_{n}}|f(z)|^{2}\left(1-|z|^{2}\right)^{l} d v(z)
$$

The following lemma is well known (cf. [13]). 
Lemma 2.21. Let $T$ be a bounded linear operator on $L_{a}^{2}\left(\mathbb{B}_{n}\right)$. If there exists a constant $C>0$ such that

$$
\|T f\|^{2} \leq C\|f\|_{L_{a, 1}^{2}}^{2}, \quad \forall f \in L_{a}^{2}\left(\mathbb{B}_{n}\right)
$$

then $T \in \mathcal{C}_{p}$ for all $p>2 n$.

\section{Asymptotic Stable Division Property and Essential Normality}

By Lemma 2.1, in order to show that a submodule $\mathcal{P}$ is $p$-essentially normal, $\forall p>n$, one needs to show that $Q M_{z_{i}}^{*} P$ is in $\mathcal{C}_{p}$ for any $p>2 n$. That means, for $f \in \mathcal{P}$, one needs to find an element in $\mathcal{P}$ that is close enough to $M_{z_{i}}^{*} f$. In the case when $\mathcal{P}$ is principal with generator $h$, the set of functions $\left\{h f: f \in \mathbb{C}\left[z_{1}, \cdots, z_{n}\right]\right\}$ is dense in $\mathcal{P}$. For a function $h f$, a reasonable approximation of $M_{z_{i}}^{*}(h f)$ will be $h M_{z_{i}}^{*} f$ (cf. [9] [8] [13] [15]). In general, suppose $\mathcal{P}$ is generated by $\left\{h_{1}, \cdots, h_{k}\right\}$, it may happen that $\sum_{j=1}^{k} h_{j} f_{j}$ equals 0 while $\sum_{j=1}^{k} h_{j} M_{z_{i}}^{*} f_{j}$ does not. Thus the distance between $M_{z_{i}}^{*}\left(\sum_{j=1}^{k} h_{j} f_{j}\right)$ and $\sum_{j=1}^{k} h_{j} M_{z_{i}}^{*} f_{j}$ may not be small (compared to $\left.\left\|\sum_{j=1}^{k} h_{j} f_{j}\right\|_{L_{a, 1}^{2}}\right)$.

One can avoid such problems by putting restrictions on the decomposition of $f$. In [20], Shalit considered submodules of the Drury-Arveson module, with the stable division property. For a submodule with the stable division property, one can always find a decomposition $f=$ $\sum_{j=1}^{k} h_{j} f_{j}$ with

$$
\sum_{j=1}^{k}\left\|h_{j} f_{j}\right\| \leq C\|f\|,
$$

where $C$ is a constant depending only on $\mathcal{P}$. Shalit showed that graded submodules with stable division property are essentially normal.

We propose the following definition of asymptotic stable division property.

Definition 3.1. Suppose $\mathcal{P}$ is a submodule of the Bergman module $L_{a}^{2}\left(\mathbb{B}_{n}\right)$. $\mathcal{P}$ is said to have the asymptotic stable division property if there exist an invertible operator $T$ on $\mathcal{P}$, a subset $\left\{h_{i}\right\}_{i \in \Lambda} \subset \mathcal{P}$, finite or countably infinite, and constants $C_{1}, C_{2}$, such that for any $f \in \mathcal{P}$, there exists $\left\{g_{i}\right\}_{i \in \Lambda} \subset \operatorname{Hol}\left(\mathbb{B}_{n}\right)$ with the following properties.

(1) $T f=\sum_{i \in \Lambda} h_{i} g_{i}$, where the convergence is pointwise if $\Lambda$ is countably infinite.

$$
\begin{gathered}
\int_{\mathbb{B}_{n}}\left(\sum_{i \in \Lambda}\left|h_{i}(z) g_{i}(z)\right|\right)^{2} d v(z) \leq C_{1}\|f\|_{L_{a}^{2}}^{2} \\
\int_{\mathbb{B}_{n}}\left(\sum_{i \in \Lambda}\left|h_{i}(z) g_{i}(z)\right|\right)^{2}\left(1-|z|^{2}\right) d v(z) \leq C_{2}\|f\|_{L_{a, 1}^{2}}^{2} .
\end{gathered}
$$

Similar to the case of stable division property, we have the following theorem.

Theorem 3.2. Suppose $\mathcal{P}$ is a submodule of $L_{a}^{2}\left(\mathbb{B}_{n}\right)$ with the asymptotic stable division property. If the generating functions $h_{i}$ are all defined in neighborhoods of $\overline{\mathbb{B}}_{n}$ and the sets of constants $\left\{C_{i}\right\}_{i \in \Lambda},\left\{N_{i}\right\}_{i \in \Lambda}$, determined by $h_{i}$ (as in Theorem[2.16), are bounded, then the submodule $\mathcal{P}$ is $p$-essentially normal for all $p>n$. In particular, if the generating functions $h_{i}$ are polynomials of bounded degrees, then $\mathcal{P}$ is $p$-essentially normal for all $p>n$. 
Proof. Denote $P$ the projection operator onto $\mathcal{P}$ and $Q$ the projection operator onto $\mathcal{P}^{\perp}$. By Lemma [2.1, it suffices to show that $\left[M_{z_{k}}^{*}, P\right]=Q M_{z_{k}}^{*} P$ is in $\mathcal{C}_{p}, \forall p>2 n$. Let $N=\max \left\{N_{i}\right.$ : $i \in \Lambda$. Since $C_{i}$ are uniformly bounded, by Lemma 2.12 (1), there is a constant $C$ such that inequality (2.1) holds for all $h_{i}$ with constants $C$ and $N$. Choose a positive integer $l>N$. Define

$$
M_{z_{k}}^{(l) *} f(z)=c_{l}^{-1} \int_{\mathbb{B}_{n}} \bar{w}_{k} f(w) K_{w}^{(l)}(z)\left(1-|w|^{2}\right)^{l} d v(w)
$$

where $K_{w}^{(l)}(z)=\frac{1}{(1-\langle z, w\rangle)^{n+l+1}}$ is the weighted reproducing kernel, and $c_{l}=\int_{\mathbb{B}_{n}}\left(1-|w|^{2}\right)^{l} d v(w)$. For $f \in L_{a}^{2}\left(\mathbb{B}_{n}\right)$,

$$
\begin{aligned}
& \left|M_{z_{k}}^{*} f(z)-M_{z_{k}}^{(l) *} f(z)\right| \\
= & \left|\int_{\mathbb{B}_{n}}\left(\bar{w}_{k}-\overline{z_{k}}\right) f(w) K_{w}(z) d v(w)-c_{l}^{-1} \int_{\mathbb{B}_{n}}\left(\bar{w}_{k}-\overline{z_{k}}\right) f(w) K_{w}^{(l)}(z)\left(1-|w|^{2}\right)^{l} d v(w)\right| \\
\lesssim & \int_{\mathbb{B}_{n}}|f(w)| \frac{|w-z|}{|1-\langle z, w\rangle|^{n+1}} d v(w) \\
\lesssim & \int_{\mathbb{B}_{n}}|f(w)| \frac{1}{|1-\langle z, w\rangle|^{n+1 / 2}} d v(w) .
\end{aligned}
$$

By Lemma 2.20 and Lemma 2.21, $M_{z_{k}}^{*}-M_{z_{k}}^{(l) *}$ is in $\mathcal{C}_{p}$ for any $p>2 n$.

For $f \in \mathcal{P}$, by assumption, $T f=\sum_{i \in \Lambda} h_{i} g_{i}$. Define

$$
S_{k} f(z)=\sum_{i \in \Lambda} h_{i}(z) G_{i}(z)
$$

where

$$
G_{i}(z)=c_{l}^{-1} \int \bar{w}_{k} g_{i}(w) K_{w}^{(l)}(z)\left(1-|w|^{2}\right)^{l} d v(w), \quad i \in \Lambda .
$$

For each $i \in \Lambda$, applying Theorem 2.16 , we get

$$
\begin{aligned}
\left|h_{i}(z) G_{i}(z)\right| & =c_{l}^{-1}\left|h_{i}(z) \int \bar{w}_{k} g_{i}(w) K_{w}^{(l)}(z)\left(1-|w|^{2}\right)^{l} d v(w)\right| \\
& \leq C c_{l}^{-1} \int_{\mathbb{B}_{n}} \frac{|1-\langle z, w\rangle|^{N}}{\left(1-|w|^{2}\right)^{n+N+1}} \int_{D(w, 1)}\left|h_{i}(\lambda) g_{i}(\lambda)\right| d v(\lambda) \frac{\left(1-|w|^{2}\right)^{l}}{|1-\langle z, w\rangle|^{n+l+1}} d v(w) \\
& =C c_{l}^{-1} \int_{\mathbb{B}_{n}}\left|h_{i}(\lambda) g_{i}(\lambda)\right| \int_{D(\lambda, 1)} \frac{1}{\left(1-|w|^{2}\right)^{n+1+N-l}|1-\langle z, w\rangle|^{n+1+l-N}} d v(w) d v(\lambda) \\
& \lesssim C \int_{\mathbb{B}_{n}}\left|h_{i}(\lambda) g_{i}(\lambda)\right| \frac{\left(1-|\lambda|^{2}\right)^{l-N}}{|1-\langle z, \lambda\rangle|^{n+1+l-N}} d v(\lambda) .
\end{aligned}
$$

By Lemma 2.20 and our assumption, $h_{i} G_{i}$ belongs to $L_{a}^{2}\left(\mathbb{B}_{n}\right)$. By [9, Proposition 5.5], $h_{i} G_{i}$ is in the principal submodule generated by $h_{i}$, which is contained in $\mathcal{P}$. Moreover,

$$
\sum_{i \in \Lambda}\left|h_{i}(z) G_{i}(z)\right| \lesssim C \int_{\mathbb{B}_{n}}\left(\sum_{i \in \Lambda}\left|h_{i}(\lambda) g_{i}(\lambda)\right|\right) \frac{\left(1-|\lambda|^{2}\right)^{l-N}}{|1-\langle z, \lambda\rangle|^{n+1+l-N}} d v(\lambda) .
$$

By condition (2) in Definition [3.1, the series $\sum_{i \in \Lambda} h_{i} G_{i}$ converges weakly. Therefore $S_{k} f=$ $\sum_{i \in \Lambda} h_{i} G_{i} \in \mathcal{P}$. 
Next, we show that $Q M_{z_{k}}^{(l) *} P$ is in $\mathcal{C}_{p}$ for any $p>2 n$. Once this is done, we will have $Q M_{z_{k}}^{*} P=Q\left(M_{z_{k}}^{*}-M_{z_{k}}^{(l) *}\right) P+Q M_{z_{k}}^{(l) *} P \in \mathcal{C}_{p}$, which is exactly what we need.

For any $f \in \mathcal{P}$,

$$
\begin{aligned}
& \left|M_{z_{k}}^{(l) *} T f(z)-S_{k} f(z)\right| \\
\leq & c_{l}^{-1} \sum_{i \in \Lambda} \mid \int \bar{w}_{k} h_{i}(w) g_{i}(w) K_{w}^{(l)}(z)\left(1-|w|^{2}\right)^{l} d v(w) \\
& -\int \bar{w}_{k} h_{i}(z) g_{i}(w) K_{w}^{(l)}(z)\left(1-|w|^{2}\right)^{l} d v(w) \mid \\
= & c_{l}^{-1} \sum_{i \in \Lambda} \mid \int\left(\bar{w}_{k}-\overline{z_{k}}\right) h_{i}(w) g_{i}(w) K_{w}^{(l)}(z)\left(1-|w|^{2}\right)^{l} d v(w) \\
& -\int\left(\bar{w}_{k}-\bar{z}_{k}\right) h_{i}(z) g_{i}(w) K_{w}^{(l)}(z)\left(1-|w|^{2}\right)^{l} d v(w) \mid \\
\lesssim & \sum_{i \in \Lambda} \int\left|h_{i}(w) g_{i}(w)\right| \frac{\left(1-|w|^{2}\right)^{l}}{|1-\langle z, w\rangle|^{n+1 / 2+l}} d v(w) \\
& +\sum_{i \in \Lambda} \int\left|h_{i}(z) g_{i}(w)\right| \frac{\left(1-|w|^{2}\right)^{l}}{|1-\langle z, w\rangle|^{n+1 / 2+l}} d v(w) .
\end{aligned}
$$

Again,

$$
\begin{aligned}
& \int\left|h_{i}(z) g_{i}(w)\right| \frac{\left(1-|w|^{2}\right)^{l}}{|1-\langle z, w\rangle|^{n+1 / 2+l}} d v(w) \\
\lesssim & \int \frac{|1-\langle z, w\rangle|^{N}}{\left(1-|w|^{2}\right)^{n+1+N}} \int_{D(w, 1)}\left|h_{i}(\lambda) g_{i}(\lambda)\right| d v(\lambda) \frac{\left(1-|w|^{2}\right)^{l}}{|1-\langle z, w\rangle|^{n+1 / 2+l}} d v(w) \\
\lesssim & \int\left|h_{i}(\lambda) g_{i}(\lambda)\right| \frac{\left(1-|\lambda|^{2}\right)^{l-N}}{|1-\langle z, \lambda\rangle|^{n+1 / 2+l-N}} d v(\lambda) .
\end{aligned}
$$

Thus

$$
\left|M_{z_{k}}^{(l) *} T f(z)-S_{k} f(z)\right| \lesssim \int\left(\sum_{i \in \Lambda}\left|h_{i}(w) g_{i}(w)\right|\right) \frac{\left(1-|w|^{2}\right)^{l-N}}{|1-\langle z, w\rangle|^{n+1 / 2+l-N}} d v(w) .
$$

Applying Lemma 2.20 (2) on the right-hand side, we obtain

$$
\left\|Q M_{z_{k}}^{(l) *} T f\right\|^{2} \leq\left\|M_{z_{k}}^{(l) *} T f-S_{k} f\right\|^{2} \lesssim \int\left(\sum_{i \in \Lambda}\left|h_{i}(w) g_{i}(w)\right|\right)^{2}\left(1-|w|^{2}\right) d v(w) \lesssim\|f\|_{L_{a, 1}^{2}}^{2} .
$$

By Lemma 2.21, $Q M_{z_{k}}^{(l) *} T P$ is in $\mathcal{C}_{p}$ for any $p>2 n$. Since $T$ is invertible on $\mathcal{P}$, we have $Q M_{z_{k}}^{(l) *} P=Q M_{z_{k}}^{(l) *} T P T^{-1} P \in \mathcal{C}_{p}, \forall p>2 n$. Thus $Q M_{z_{k}}^{*} P$ is in $\mathcal{C}_{p}$ for any $p>2 n$. By Lemma 2.1. $\mathcal{P}$ is $p$-essentially normal for all $p>n$. This completes the proof.

Remark 3.3. As indicated in the title, the aim of this paper is to find a unified proof that works for most known results of the Arveson-Douglas Conjecture. First, suppose $h$ is a holomorphic function defined in a neighborhood of $\overline{\mathbb{B}_{n}}$ and $\mathcal{P}_{h}$ is the principal submodule generated by $h$. Then $\mathcal{P}_{h}$ has the asymptotic stable division property trivially. Second, Theorem 3.2 generalizes Shalit's result in that we do not require the generators to be polynomials and we do not require 
the submodule to be graded. In fact, by Theorem [2.16 and Theorem 3.2, any finite set of generators $\left\{h_{i}\right\}_{i=1}^{k}$, defined in a neighborhood of $\overline{\mathbb{B}_{n}}$, satisfying inequality (3.1) for relevant norms, generate an essentially normal submodule. Finally, we will show in Theorem 4.1 that most of the submodules in [10][11][12] have the asymptotic stable division property.

Before proving Theorem 4.1, let us discuss the matter with some generality. Consider the following technical hypotheses.

Hypothesis 1: Suppose $I \subset \mathbb{C}\left[z_{1}, \cdots, z_{n}\right]$ is an ideal, $N, M$ are positive integers, $C>0$. For any $\epsilon>0$ sufficiently small, there exists $R_{0}>0$ with the following property. For $R>R_{0}$, there exist constants $0<\delta<1, C^{\prime}>0$, open covers $\left\{E_{i}\right\}_{i \in \Lambda},\left\{F_{i}\right\}_{i \in \Lambda}$, finite or countably infinite, of $\mathbb{B}_{\delta}:=\left\{z \in \mathbb{B}_{n}:|z|>\delta\right\}$, and for each $i \in \Lambda$, a subset $\left\{p_{i j}\right\}_{j \in \Gamma_{i}} \subseteq I$, finite or countably infinite, such that the following hold.

(1) $E_{i} \subset F_{i} \subset \mathbb{B}_{n}$.

(2) For $k \in \mathbb{N}$, denote

$$
E_{i k}=\left\{w \in \mathbb{B}_{n}: \beta\left(w, E_{i}\right)<k R\right\} .
$$

Then $E_{i 3} \subset F_{i}$. Moreover, any $z \in \mathbb{B}_{n}$ belongs to at most $M$ of the sets $\left\{F_{i}\right\}_{i \in \Lambda}$.

(3) For any $i \in \Lambda, j \in \Gamma_{i}, \operatorname{deg} p_{i j}<N$.

(4) For any $i \in \Lambda$ and any $f \in \mathcal{P}_{I}$, there exist $\left\{f_{i j}\right\}_{j \in \Gamma_{i}} \subseteq \operatorname{Hol}\left(E_{i 3}\right)$ such that

$$
\int_{E_{i 3}}\left|\sum_{j \in \Gamma_{i}} p_{i j}(\lambda) f_{i j}(\lambda)-f(\lambda)\right|^{2} d v(\lambda) \leq \epsilon \int_{F_{i}}|f(\lambda)|^{2} d v(\lambda) .
$$

$$
\int_{E_{i 3}}\left(\sum_{j \in \Gamma_{i}}\left|p_{i j}(\lambda) f_{i j}(\lambda)\right|\right)^{2} d v(\lambda) \leq C \int_{F_{i}}|f(\lambda)|^{2} d v(\lambda) .
$$

(iii)

$$
\begin{aligned}
& \int_{E_{i 3}}\left(\sum_{j \in \Gamma_{i}}\left|p_{i j}(\lambda) f_{i j}(\lambda)\right|\right)^{2}\left(1-|\lambda|^{2}\right) d v(\lambda) \\
\leq & C^{\prime} \int_{F_{i}}|f(\lambda)|^{2}\left(1-|\lambda|^{2}\right) d v(\lambda) .
\end{aligned}
$$

(iv) The maps $f \mapsto f_{i j}$ are linear.

Theorem 3.4. Under Hypothesis 1, $\mathcal{P}_{I}$ has the asymptotic stable division property with generating functions consisting of polynomials of bounded degrees.

Combining Theorem 3.2 and Theorem 3.4, we have the following corollary.

Corollary 3.5. Assume Hypothesis 1. Then the submodule $\mathcal{P}_{I}$ is p-essentially normal for all $p>n$.

Proof of Theorem 3.4. Let $I, N, M, C$ be as in Hypothesis 1. Let $\epsilon>0, R>r>0$ be determined later. We will always assume that $R>R_{0}$, where $R_{0}$ is determined by $\epsilon$, as in Hypothesis 1. For $i \in \Lambda$, define

$$
\varphi_{i}(z)=\left\{\begin{array}{lc}
1-\frac{\beta\left(z, E_{i}\right)}{R}, & \beta\left(z, E_{i}\right) \leq R \\
0, & \text { otherwise }
\end{array}\right.
$$

It is easy to see that 
(1) $0 \leq \varphi_{i} \leq 1$.

(2) $\left\{z: \varphi_{i}(z) \neq 0\right\}=E_{i 1}$.

(3) $\left|\varphi_{i}(z)-\varphi_{i}(w)\right| \leq \frac{\beta(z, w)}{R}$.

Fix a positive integer $l>N$. Define the linear operator

$$
\hat{T}: I \rightarrow \mathcal{P}_{I}, f \mapsto \hat{T} f(z)=\sum_{i \in \Lambda, j \in \Gamma_{i}} p_{i j}(z) g_{i j}(z)
$$

where

$$
g_{i j}(z)=\int_{\mathbb{B}_{n}} \varphi_{i}(w) f_{i j}(w) K_{w}^{(l)}(z)\left(1-|w|^{2}\right)^{l} d v(w) .
$$

First, we show that $\hat{T}$ is well-defined and extends to a bounded operator on $\mathcal{P}_{I}$. For each pair $i, j$ and any $z \in \mathbb{B}_{n}$,

$$
\begin{aligned}
& \left|p_{i j}(z) g_{i j}(z)\right| \\
\leq & \left|p_{i j}(z)\right| \int \varphi_{i}(w)\left|f_{i j}(w)\right| \frac{\left(1-|w|^{2}\right)^{l}}{|1-\langle z, w\rangle|^{n+1+l}} d v(w) \\
\lesssim & \int_{E_{i 1}} \frac{|1-\langle z, w\rangle|^{N}}{\left(1-|w|^{2}\right)^{n+1+N}} \int_{D(w, 1)}\left|p_{i j}(\lambda) f_{i j}(\lambda)\right| d v(\lambda) \frac{\left(1-|w|^{2}\right)^{l}}{|1-\langle z, w\rangle|^{n+1+l}} d v(w) \\
\lesssim & \int_{E_{i 2}}\left|p_{i j}(\lambda) f_{i j}(\lambda)\right| \frac{\left(1-|\lambda|^{2}\right)^{l-N}}{|1-\langle z, \lambda\rangle|^{n+1+l-N}} d v(\lambda) .
\end{aligned}
$$

Here the second inequality comes from Theorem 2.17. Hence

$$
\left\|p_{i j} g_{i j}\right\|^{2} \lesssim \int_{E_{i 2}}\left|p_{i j} f_{i j}\right|^{2} d v \leq C\|f\|^{2} .
$$

Therefore the map $f \rightarrow p_{i j} g_{i j}$ extends to a bounded linear operator from $\mathcal{P}_{I}$ to the principal submodule generated by $p_{i j}$. By [9, Proposition 5.5], the image for any $f \in \mathcal{P}_{I}$ equals $p_{i j} g_{i j}$ for some $g_{i j} \in \operatorname{Hol}\left(\mathbb{B}_{n}\right)$.

Next, we show that $\hat{T}$ itself extends to a bounded linear operator. From inequality (3.2), we see that

$$
\begin{aligned}
\sum_{i, j}\left|p_{i j}(z) g_{i j}(z)\right| & \lesssim \sum_{i, j} \int_{E_{i 2}}\left|p_{i j}(\lambda) f_{i j}(\lambda)\right| \frac{\left(1-|\lambda|^{2}\right)^{l-N}}{|1-\langle z, \lambda\rangle|^{n+1+l-N}} d v(\lambda) \\
& =\int_{\mathbb{B}_{n}}\left(\sum_{i} \chi_{E_{i 2}}(\lambda) \sum_{j}\left|p_{i j}(\lambda) f_{i j}(\lambda)\right|\right) \frac{\left(1-|\lambda|^{2}\right)^{l-N}}{|1-\langle z, \lambda\rangle|^{n+1+l-N}} d v(\lambda)
\end{aligned}
$$

By Lemma 2.20 (1),

$$
\begin{aligned}
\|\hat{T} f\|^{2} & \leq\left\|\sum_{i, j}\left|p_{i j} g_{i j}\right|\right\|^{2} \\
& \lesssim \int_{\mathbb{B}_{n}}\left(\sum_{i} \chi_{E_{i 2}}(\lambda) \sum_{j}\left|p_{i j}(\lambda) f_{i j}(\lambda)\right|\right)^{2} d v(\lambda) .
\end{aligned}
$$


By our hypotheses, for each $\lambda$, there are at most $M$ functions $\chi_{E_{i 2}}$ with $\chi_{E_{i 2}}(\lambda) \neq 0$. By Lemma 2.18 ,

$$
\left(\sum_{i} \chi_{E_{i 2}}(\lambda) \sum_{j}\left|p_{i j}(\lambda) f_{i j}(\lambda)\right|\right)^{2} \leq M \sum_{i} \chi_{E_{i 2}}(\lambda)\left(\sum_{j}\left|p_{i j}(\lambda) f_{i j}(\lambda)\right|\right)^{2} .
$$

Therefore

$$
\begin{aligned}
\|\hat{T} f\|^{2} \leq\left\|\sum_{i, j}\left|p_{i j} g_{i j}\right|\right\|^{2} & \lesssim M \sum_{i} \int_{E_{i 2}}\left(\sum_{j}\left|p_{i j}(\lambda) f_{i j}(\lambda)\right|\right)^{2} d v(\lambda) \\
& \leq C M \sum_{i} \int_{F_{i}}|f(\lambda)|^{2} d v(\lambda) \\
& \leq C M^{2}\|f\|^{2}
\end{aligned}
$$

Hence $\hat{T}$ defines a bounded operator.

Arguments similar to those in the proof of Theorem 3.2 show that $\hat{T} f \in \mathcal{P}_{I}$ for all $f \in I$.

To sum it up, $\hat{T}$ extends to a bounded linear operator on $\mathcal{P}_{I}$ and for each $f \in \mathcal{P}_{I}$, $\hat{T} f$ has the form $\sum_{i, j} p_{i j} g_{i j}$. Moreover,

$$
\left\|\sum_{i, j}\left|p_{i j} g_{i j}\right|\right\|^{2} \lesssim C M^{2}\|f\|^{2}
$$

Since $l>N$, by Lemma 2.20, the estimates above will also give us the following:

$$
\int\left(\sum_{i, j}\left|p_{i j}(z) g_{i j}(z)\right|\right)^{2}\left(1-|z|^{2}\right) d v(z) \lesssim C^{\prime} M^{2}\|f\|_{L_{a, 1}^{2}}^{2}
$$

Next, we want to show that inequality (1) in Definition 3.1 holds. We will obtain this by showing that a finite rank perturbation of $\hat{T}$ is invertible on $\mathcal{P}_{I}$. For any $z \in \mathbb{B}_{n}$ and the chosen $r<R$,

$$
\begin{aligned}
& \left|\hat{T} f(z)-\sum_{i, j} p_{i j}(z) \int_{D(z, r)} \varphi_{i}(w) f_{i j}(w) K_{w}^{(l)}(z)\left(1-|w|^{2}\right)^{l} d v(w)\right| \\
\leq & \sum_{i, j}\left|p_{i j}(z)\right| \int_{E_{i 1} \backslash D(z, r)}\left|f_{i j}(w)\right| \frac{\left(1-|w|^{2}\right)^{l}}{|1-\langle z, w\rangle|^{n+1+l}} d v(w) \\
\lesssim & \sum_{i, j} \int_{E_{i 1} \backslash D(z, r)} \frac{|1-\langle z, w\rangle|^{N}}{\left(1-|w|^{2}\right)^{n+1+N}} \int_{D(w, 1)}\left|p_{i j}(\lambda) f_{i j}(\lambda)\right| d v(\lambda) \frac{\left(1-|w|^{2}\right)^{l}}{|1-\langle z, w\rangle|^{n+1+l}} d v(w) \\
\lesssim & \sum_{i, j} \int_{E_{i 2} \backslash D(z, r-1)}\left|p_{i j}(\lambda) f_{i j}(\lambda)\right| \frac{\left(1-|\lambda|^{2}\right)^{l-N}}{|1-\langle z, \lambda\rangle|^{n+1+l-N}} d v(\lambda) \\
= & \int_{D(z, r-1)^{c}}\left(\sum_{i, j} \chi_{E_{i 2}}(\lambda)\left|p_{i j}(\lambda) f_{i j}(\lambda)\right|\right) \frac{\left(1-|\lambda|^{2}\right)^{l-N}}{|1-\langle z, \lambda\rangle|^{n+1+l-N}} d v(\lambda) .
\end{aligned}
$$


Also,

$$
\begin{aligned}
& \mid \sum_{i, j} p_{i j}(z) \int_{D(z, r)} \varphi_{i}(w) f_{i j}(w) K_{w}^{(l)}(z)\left(1-|w|^{2}\right)^{l} d v(w) \\
& -\sum_{i, j} p_{i j}(z) \varphi_{i}(z) \int_{D(z, r)} f_{i j}(w) K_{w}^{(l)}(z)\left(1-|w|^{2}\right)^{l} d v(w) \mid \\
\leq & \sum_{i, j}\left|p_{i j}(z)\right| \int_{E_{i 2}} \frac{r}{R}\left|f_{i j}(w)\right| \frac{\left(1-|w|^{2}\right)^{l}}{|1-\langle z, w\rangle|^{n+1+l}} d v(w) \\
\lesssim & \frac{r}{R} \sum_{i, j} \int_{E_{i 2}} \frac{|1-\langle z, w\rangle|^{N}}{\left(1-|w|^{2}\right)^{n+1+N}} \int_{D(w, 1)}\left|p_{i j}(\lambda) f_{i j}(\lambda)\right| d v(\lambda) \frac{\left(1-|w|^{2}\right)^{l}}{|1-\langle z, w\rangle|^{n+1+l}} d v(w) \\
\lesssim & \frac{r}{R} \sum_{i, j} \int_{E_{i 3}}\left|p_{i j}(\lambda) f_{i j}(\lambda)\right| \frac{\left(1-|\lambda|^{2}\right)^{l-N}}{|1-\langle z, \lambda\rangle|^{n+1+l-N}} d v(\lambda) \\
= & \frac{r}{R} \int\left(\sum_{i, j} \chi_{E_{i 3}}(\lambda)\left|p_{i j}(\lambda) f_{i j}(\lambda)\right|\right) \frac{\left(1-|\lambda|^{2}\right)^{l-N}}{|1-\langle z, \lambda\rangle|^{n+1+l-N}} d v(\lambda) .
\end{aligned}
$$

Finally, notice that if $\varphi_{i}(z) \neq 0$, then $z \in E_{i 1}$. By Lemma 2.20 (3),

$$
\sum_{i, j} p_{i j}(z) \varphi_{i}(z) \int_{D(z, r)} f_{i j}(w) K_{w}^{(l)}(z)\left(1-|w|^{2}\right)^{l} d v(w)=c_{r, l} \sum_{i, j} \varphi_{i}(z) p_{i j}(z) f_{i j}(z) .
$$

By Lemma 2.20 and the previous arguments,

$$
\left\|\hat{T} f-c_{r, l} \sum_{i} \varphi_{i} \sum_{j} p_{i j} f_{i j}\right\| \lesssim\left(\epsilon_{r-1, l-N}+\frac{r}{R}\right)\left\|\sum_{i, j} \chi_{E_{i 3}}\left|p_{i j} f_{i j}\right|\right\| \lesssim\left(\epsilon_{r-1, l-N}+\frac{r}{R}\right)(C M)^{1 / 2}\|f\| .
$$

On the other hand, by Hypothesis 1,

$$
\int_{E_{i 3}}\left|\sum_{j} p_{i j}(\lambda) f_{i j}(\lambda)-f(\lambda)\right|^{2} d v(\lambda) \leq \epsilon \int_{F_{i}}|f(\lambda)|^{2} d v(\lambda) .
$$

So

$$
\begin{aligned}
& \int_{\mathbb{B}_{n}}\left|\sum_{i} \varphi_{i}(\lambda) \sum_{j} p_{i j}(\lambda) f_{i j}(\lambda)-\sum_{i} \varphi_{i}(\lambda) f(\lambda)\right|^{2} d v(\lambda) \\
\leq & M \sum_{i} \int_{E_{i 1}}\left|\sum_{j} p_{i j}(\lambda) f_{i j}(\lambda)-f(\lambda)\right|^{2} d v(\lambda) \\
\leq & \epsilon M \sum_{i} \int_{F_{i}}|f(\lambda)|^{2} d v(\lambda) \\
\leq & \epsilon M^{2}\|f\|^{2} .
\end{aligned}
$$

Combining the above estimates, we get

$$
\left\|\hat{T} f-c_{r, l} \sum_{i} \varphi_{i} f\right\| \lesssim\left[\left(\epsilon_{r-1, l-N}+\frac{r}{R}\right)(C M)^{1 / 2}+\epsilon^{1 / 2} M\right]\|f\| .
$$


Let $T_{\sum_{i} \varphi_{i}}$ be the Toeplitz operator with symbol $\sum_{i} \varphi_{i}$. Then the above implies

$$
\left\|\hat{T} f-c_{r, l} T_{\sum_{i} \varphi_{i}} f\right\| \lesssim\left[\left(\epsilon_{r-1, l-N}+\frac{r}{R}\right)(C M)^{1 / 2}+\epsilon^{1 / 2} M\right]\|f\| .
$$

Since $C, l, N$ and $M$ are fixed, we can choose $\epsilon, r$, and then $R>R_{0}$ so that

$$
\left(\epsilon_{r-1, l-N}+\frac{r}{R}\right)(C M)^{1 / 2}+\epsilon^{1 / 2} M<1 / 2 c_{r, l} .
$$

For a positive integer $k$, denote

$$
I_{k}=\{q \in I, \operatorname{deg} q \leq k\} .
$$

Since $\sum \varphi_{i} \geq \chi_{\mathbb{B}_{\delta}}$, we can choose $k$ large enough so that the compression of $T_{\sum_{i} \varphi_{i}}$ on $\mathcal{P}_{I} \ominus I_{k}$ is invertible on $\mathcal{P}_{I} \ominus I_{k}$ and the norm of its inverse is less than 2. Then the compression of $\hat{T}$ on $\mathcal{P}_{I} \ominus I_{k}$ is invertible. By a block matrix argument, it is easy to see that $T:=\hat{T}\left(P-P_{I_{k}}\right)+P_{I_{k}}$ is invertible on $\mathcal{P}_{I}$. Here $P$ denotes the orthogonal projection onto $\mathcal{P}_{I}$. Choose an orthonormal basis $\left\{h_{i}\right\}$ for $I_{k}$ under the Bergman norm. For any $f \in \mathcal{P}_{I}$,

$$
T f=\sum_{i, j} p_{i j} g_{i j}+P_{I_{k}} f=\sum_{i, j} p_{i j} g_{i j}+\sum_{i} c_{i} h_{i} .
$$

Then by inequalities (3.3) and (3.4),

$$
\int\left(\sum_{i, j}\left|p_{i j} g_{i j}\right|\right)^{2} d v \lesssim\left\|\left(P-P_{I_{k}}\right) f\right\|^{2} \leq\|f\|^{2} .
$$

Since $I_{k}$ is finitely dimensional, we also have

$$
\int\left(\sum_{i}\left|c_{i} h_{i}\right|\right)^{2} d v \lesssim\left\|\sum_{i} c_{i} h_{i}\right\|^{2} \lesssim\|f\|^{2} .
$$

It is also easy to see that $P_{I_{k}}$ extends to a bounded operator on the closure of $I$ in $L_{a, 1}^{2}\left(\mathbb{B}_{n}\right)$. Therefore

$$
\int\left(\sum_{i}\left|c_{i} h_{i}\right|\right)^{2}\left(1-|z|^{2}\right) d v \lesssim\|f\|_{L_{a, 1}^{2}}^{2}
$$

and

$$
\int\left(\sum_{i, j}\left|p_{i j} g_{i j}\right|\right)^{2}\left(1-|z|^{2}\right) d v \lesssim\left\|f-P_{I_{k}} f\right\|_{L_{a, 1}^{2}}^{2} \lesssim\|f\|_{L_{a, 1}^{2}}^{2} .
$$

This completes the proof.

\section{A Distance Estimate}

As promised in Remark 3.3, we are going to show that most submodules in the known results of the Geometric Arveson-Douglas Conjecture has the asymptotic stable division property. In fact, we will prove the following more general result.

Theorem 4.1. Suppose $I$ is an ideal in $\mathbb{C}\left[z_{1}, \cdots, z_{n}\right]$ with primary decomposition $I=\cap_{j=1}^{k} I_{j}^{m_{j}}$, where $I_{j}$ are prime ideals. Assume the following.

(1) For any $j=1, \cdots, k, Z\left(I_{j}\right)$ has no singular points on $\partial \mathbb{B}_{n}$ and intersects $\partial \mathbb{B}_{n}$ transversely.

(2) Any pair of the varieties $\left\{Z\left(I_{j}\right)\right\}$ does not intersect on $\partial \mathbb{B}_{n}$. 
Then I satisfies Hypothesis 1. Consequently, the submodule $\mathcal{P}_{I}$ has the asymptotic stable division property with generating functions being polynomials of uniformly bounded degrees, and $\mathcal{P}_{I}$ is p-essentially normal for all $p>n$.

We will prove Theorem 4.1 in the remaining sections.

Notations: For the remainder of this paper, we reserve the notations $I, m_{j}$ and $I_{j}$ for the ones mentioned in Theorem 4.1. Denote $Z=Z(I), Z_{j}=Z\left(I_{j}\right), \mathcal{Z}=Z \cap \mathbb{B}_{n}$ and $\mathcal{Z}_{j}=Z_{j} \cap \mathbb{B}_{n}$.

As a preparation, we will prove a distance estimate for $Z(I)$ in this section.

Proposition 4.2. For fixed $R>0$,

$$
\sup _{w \in D(z, R) \cap Z} \beta\left(w, T_{z} Z+z\right) \rightarrow 0, \quad|z| \rightarrow 1, z \in \mathcal{Z} .
$$

Here $T_{z} Z$ is the complex tangent space of $Z$ at $z$, viewed as a linear subspace of $\mathbb{C}^{n}$.

Proof. Since $Z$ intersects $\partial \mathbb{B}_{n}$ transversely, there exists $C_{1}>0$ such that, for any $z \in Z$ close enough to $\partial \mathbb{B}_{n}$, there exists a non-zero vector $v_{z} \in T_{z} Z$ such that

$$
\left|Q_{z}\left(v_{z}\right)\right| \leq C_{1}\left|\left\langle v_{z}, z\right\rangle\right| \text {. }
$$

It is also easy to see that there exists $C_{2}>0$ such that for $r>0$ sufficiently small and any $z \in Z$ close enough to $\partial \mathbb{B}_{n}$,

$$
\sup _{w \in Z \cap B(z, r)} \operatorname{dist}\left(w, T_{z} Z+z\right) \leq C_{2} r^{2} .
$$

Here we use "dist" to denote the Euclidean distance. Also, since $R>0$ is fixed, there exists $C_{3}>0$ such that for any $z, w \in \mathbb{B}_{n}$ and $\beta(z, w)<R$,

$$
\left|P_{z}(w-z)\right| \leq C_{3}\left(1-|z|^{2}\right),|w-z|^{2} \leq C_{3}\left(1-|z|^{2}\right) .
$$

For any $z \in Z$ and $w \in D(z, R) \cap Z$, let $\lambda \in T_{z} Z$ be such that

$$
\operatorname{dist}(w, \lambda+z)=\operatorname{dist}\left(w, T_{z} Z+z\right) \text {. }
$$

Then

$$
|w-(\lambda+z)| \leq C_{2}|w-z|^{2} \leq C_{2} C_{3}\left(1-|z|^{2}\right)
$$

Let $\lambda^{\prime}=\lambda-\frac{\langle\lambda+z-w, z\rangle}{\left\langle v_{z}, z\right\rangle} v_{z}$. Then $\lambda^{\prime} \in T_{z} Z$. We need to estimate $\beta\left(\lambda^{\prime}+z, w\right)$. Noticing that $\left\langle\lambda^{\prime}+z, z\right\rangle=\langle w, z\rangle$, we have

$$
\left|\varphi_{z}\left(\lambda^{\prime}+z\right)-\varphi_{z}(w)\right|=\frac{\left(1-|z|^{2}\right)^{1 / 2}\left|Q_{z}\left(w-\lambda^{\prime}\right)\right|}{|1-\langle w, z\rangle|} \lesssim \frac{\left|Q_{z}\left(w-\lambda^{\prime}\right)\right|}{\left(1-|z|^{2}\right)^{1 / 2}} .
$$

Since

$$
\begin{aligned}
\left|Q_{z}\left(w-\lambda^{\prime}\right)\right| & \leq\left|Q_{z}(w-\lambda)\right|+\frac{|\langle\lambda+z-w, z\rangle|}{\left|\left\langle v_{z}, z\right\rangle\right|}\left|Q_{z}\left(v_{z}\right)\right| \\
& =\left|Q_{z}(w-(\lambda+z))\right|+\frac{|\langle\lambda+z-w, z\rangle|}{\left|\left\langle v_{z}, z\right\rangle\right|}\left|Q_{z}\left(v_{z}\right)\right| \\
& \leq\left(1+C_{1}\right)|w-(\lambda+z)| \\
& \leq\left(1+C_{1}\right) C_{2} C_{3}\left(1-|z|^{2}\right)
\end{aligned}
$$

we have

$$
\left|\varphi_{z}\left(\lambda^{\prime}+z\right)-\varphi_{z}(w)\right| \lesssim\left(1-|z|^{2}\right)^{1 / 2}
$$


Since $w \in D(z, R)$, from the above inequality, for $z$ close enough to $\partial \mathbb{B}_{n}, \varphi_{z}(w), \varphi_{z}\left(\lambda^{\prime}+z\right)$ fall into a compact subset of $\mathbb{B}_{n}$. Hence

$$
\beta\left(\lambda^{\prime}+z, w\right)=\beta\left(\varphi_{z}\left(\lambda^{\prime}+z\right), \varphi_{z}(w)\right) \approx\left|\varphi_{z}\left(\lambda^{\prime}+z\right)-\varphi_{z}(w)\right| \lesssim\left(1-|z|^{2}\right)^{1 / 2} .
$$

This completes the proof.

It is convenient to consider the following modified version of tangent space, because it is invariant under the Möbius transform $\varphi_{z}$.

Definition 4.3. For $z \in \mathcal{Z}$, let us define the normal tangent cone $N T_{z} Z$ to be

$$
N T_{z} Z=T_{z} Z \cap z^{\perp}+\mathbb{C} z .
$$

Note that $z \in N T_{z} Z$.

Lemma 4.4. For any $R>0$, we have

$$
\sup _{w \in\left(T_{z} Z+z\right) \cap D(z, R)} \beta\left(w, N T_{z} Z\right) \rightarrow 0, \quad z \in \mathcal{Z},|z| \rightarrow 1 .
$$

Proof. For $z \in \mathcal{Z}$ close enough to $\partial \mathbb{B}_{n}$, let $z_{0}=P_{T_{z} Z} z$. Since $\mathcal{Z}$ intersects $\partial \mathbb{B}_{n}$ transversely, we can assume that $\left|z_{0}\right| \geq \epsilon$ for some $0<\epsilon<1$.

Suppose $w \in T_{z} Z$ and $\beta(w+z, z)<R$. Let $\lambda=P_{z} w+w-\langle w, z\rangle \frac{z_{0}}{\left|z_{0}\right|^{2}}$. Then $\lambda \in N T_{z} Z$ and $\langle w, z\rangle=\langle\lambda, z\rangle$. So $\varphi_{z}(\lambda+z)$ is well defined. Therefore

$$
\left|\varphi_{z}(z+w)-\varphi_{z}(z+\lambda)\right|=\left|\frac{\left(1-|z|^{2}\right)^{1 / 2} \frac{\langle w, z\rangle}{\left|z_{0}\right|^{2}} Q_{z}\left(z_{0}\right)}{1-\langle z+w, z\rangle}\right| \lesssim \frac{\frac{1-|z|^{2}}{\left|z_{0}\right|}}{\left(1-|z|^{2}\right)^{1 / 2}} \leq \epsilon^{-1}\left(1-|z|^{2}\right)^{1 / 2} \rightarrow 0,
$$

where the first inequality follows from the fact that $|\langle w, z\rangle|=|\langle w+z-z, z\rangle| \lesssim 1-|z|^{2}$. This completes the prove.

Combining Proposition 4.2 Lemma 4.4, we have the following lemma.

Lemma 4.5. For any $R>0$, we have

$$
\sup _{w \in Z \cap D(z, R)} \beta\left(w, N T_{z} Z\right) \rightarrow 0, \quad z \in \mathcal{Z},|z| \rightarrow 1 .
$$

\section{A Covering Lemma}

As a first step to proving Theorem 4.1, for arbitrarily large $R>0$, we will construct covers $\left\{E_{i}\right\}_{i \in \Lambda}$ and $\left\{F_{i}\right\}_{i \in \Lambda}$. In [24], Xia constructed covers of $\mathbb{B}_{n}$ with the bounded overlap condition (2) in Hypothesis 1. Our construction will follow the general framework of [24], but with additional requirements. First, our covers need to take the variety $\mathcal{Z}$ into account. For this reason, we will construct the covers in two steps, first on $\mathcal{Z}$ and then on $\mathbb{B}_{n}$. Second, to construct the decompositions on each cover set, we need the sets to be rotation invariant in certain directions. This property will be used in the proof of Lemma 6.4.

For an arbitrarily large $R>0$, choose $s^{\prime}$ so that

$$
\left(s^{\prime}-1\right) \log 2 \leq 16 R<s^{\prime} \log 2,
$$

and then take $s=10 s^{\prime}$. For a positive integer $k$, define

$$
r_{k}=\sqrt{1-2^{-2 s k}}, r_{k}^{\prime}=\sqrt{1-2^{-2 s^{\prime} k}}
$$

Write $\mathcal{Z}_{k}=r_{k} S \cap \mathcal{Z}$, where $r_{k} S=\left\{r_{k} x: x \in S\right\}$. Let $\mathcal{L}_{k} \subset \mathcal{Z}_{k}$ be maximal with respect to the following property: if $u_{i}, u_{j} \in \mathcal{L}_{k}$ and $u_{i} \neq u_{j}$, then $d_{S}\left(u_{i}, u_{j}\right)>s 2^{-s k-2}$. 
For $u \in \mathcal{L}_{k}$, set

$$
\begin{aligned}
& \tilde{E}_{u}=\left\{z \in \mathbb{B}_{n}: 1-|z|^{2}>2^{-5 s},|1-\langle z, u\rangle|>s^{-2}, \frac{1-|z|^{2}}{|1-\langle z, u\rangle|^{2}}<2^{-2 s}\right\}, \\
& \tilde{F}_{u}=\left\{z \in \mathbb{B}_{n}: 1-|z|^{2}>2^{-6 s},|1-\langle z, u\rangle|>\frac{1}{2} s^{-2}, \frac{1-|z|^{2}}{|1-\langle z, u\rangle|^{2}}<2^{-s}\right\},
\end{aligned}
$$

and

$$
E_{u}=\varphi_{u}\left(\tilde{E}_{u}\right), \quad F_{u}=\varphi_{u}\left(\tilde{F}_{u}\right)
$$

Let

$$
\mathcal{O}=\left\{w \in \mathbb{B}_{n}: \beta(w, \mathcal{Z})>5 s^{\prime} \log 2\right\} .
$$

Take $S_{k}=r_{k}^{\prime} S \cap \mathcal{O}$. Let $\mathcal{L}_{k}^{\prime} \subseteq S_{k}$ be maximal with respect to the following property: if $v_{i}, v_{j} \in S_{k}$ and $v_{i} \neq v_{j}$, then $d_{S}\left(v_{i}, v_{j}\right)>s^{\prime} 2^{-s^{\prime} k-2}$. For $v \in \mathcal{L}_{k}^{\prime}$, let

$$
\tilde{E}_{v}^{\prime}=\left\{z \in \mathbb{B}_{n}: 1-|z|^{2}>2^{-5 s^{\prime}},|1-\langle z, v\rangle|>s^{\prime-2}, \frac{1-|z|^{2}}{|1-\langle z, u\rangle|^{2}}<2^{-2 s^{\prime}}\right\},
$$

and

$$
E_{v}^{\prime}=\varphi_{v}\left(\tilde{E}_{v}^{\prime}\right)
$$

Set

$$
\tilde{F}_{v}^{\prime}=\tilde{E}_{v 3}^{\prime}, \quad F_{v}^{\prime}=E_{v 3}^{\prime},
$$

where for any set $A \subset \mathbb{B}_{n}$ and $j \in \mathbb{N}$, we denote $A_{j}=\left\{w \in \mathbb{B}_{n}: \beta(w, A)<j R\right\}$. Let $K$ be a positive integer determined later, and $\mathcal{L}=\cup_{k \geq K} \mathcal{L}_{k}, \mathcal{L}^{\prime}=\cup_{k \geq 10 K} \mathcal{L}_{k}^{\prime}$.

Let us establish some basic properties of the sets.

Lemma 5.1. For $k, s^{\prime}$ large enough and $u \in \mathcal{L}_{k}, v \in \mathcal{L}_{k}^{\prime}$, the following hold.

$$
\begin{aligned}
& E_{u}=\left\{w \in \mathbb{B}_{n}: 1-|w|^{2} \in\left(2^{-2(k+3) s}, 2^{-2(k+1) s}\right), d(u, w)<s 2^{-k s}, 1-\left|\varphi_{u}(w)\right|^{2}>2^{-5 s}\right\}, \\
& E_{v}^{\prime}=\left\{w \in \mathbb{B}_{n}: 1-|w|^{2} \in\left(2^{-2(k+3) s^{\prime}}, 2^{-2(k+1) s^{\prime}}\right), d(v, w)<s^{\prime} 2^{-k s^{\prime}}, 1-\left|\varphi_{v}(w)\right|^{2}>2^{-5 s^{\prime}}\right\}, \\
& F_{u}=\left\{w \in \mathbb{B}_{n}: 1-|w|^{2} \in\left(2^{-2(k+4) s}, 2^{-2 k s-s}\right), d(u, w)<\sqrt{2} s 2^{-k s} .1-\left|\varphi_{u}(w)\right|^{2}>2^{-6 s}\right\},
\end{aligned}
$$

$$
E_{u} \subseteq D(u, 3 s \log 2), \quad E_{v}^{\prime} \subseteq D\left(v, 3 s^{\prime} \log 2\right), \quad F_{u} \subset D(u, 4 s \log 2)
$$

Thus $E_{u 4} \subset F_{u}$.

$$
\left\{w \in \mathbb{B}_{n}: \beta\left(w, E_{u}\right)<\frac{s}{4} \log 2\right\} \subset F_{u}
$$

$$
\begin{gathered}
E_{u} \supseteq\left\{w \in \mathbb{B}_{n}: 1-|w|^{2} \in\left[2^{-2(k+2) s}, 2^{-2(k+1) s}\right), d_{S}(u, w)<s 2^{-k s-1}\right\}, \\
E_{v}^{\prime} \supseteq\left\{w \in \mathbb{B}_{n}: 1-|w|^{2} \in\left[2^{-2(k+2) s^{\prime}}, 2^{-2(k+1) s^{\prime}}\right), d_{S}(v, w)<s^{\prime} 2^{-k s^{\prime}-1}\right\} .
\end{gathered}
$$

(5) There exists a constant $M>0$, depending only on $n$, such that any $z \in \mathbb{B}_{n}$ belongs to at most $M$ of the sets $\left\{F_{u}\right\}_{u \in \mathcal{L}} \cup\left\{F_{v}^{\prime}\right\}_{v \in \mathcal{L}^{\prime}}$. 
Proof. First, we prove (1). Suppose $u \in \mathcal{L}_{k}$. By definition, $w \in E_{u}$ if and only if $\varphi_{u}(w) \in \tilde{E}_{u}$, i.e.,

$$
1-\left|\varphi_{u}(w)\right|^{2}>2^{-5 s}, \quad\left|1-\left\langle\varphi_{u}(w), u\right\rangle\right|>s^{-2}, \quad \frac{1-\left|\varphi_{u}(w)\right|^{2}}{\left|1-\left\langle\varphi_{u}(w), u\right\rangle\right|^{2}}<2^{-2 s} .
$$

Since $1-|u|^{2}=2^{-2 s k}$ and by Lemma 2.9 (1), the conditions above are equivalent to

$$
1-\left|\varphi_{u}(w)\right|^{2}>2^{-5 s}, \quad|1-\langle w, u\rangle|<s^{2} 2^{-2 s k}, \quad 1-|w|^{2}<2^{-2(k+1) s} .
$$

Also, by Lemma $2.12(2)$,

$$
1-|w|^{2}=1-\left|\varphi_{u} \varphi_{u}(w)\right|^{2}>\frac{1}{4}\left(1-|u|^{2}\right)\left(1-\left|\varphi_{u}(w)\right|^{2}\right)>\frac{1}{4} 2^{-2 s k-5 s}>2^{-2(k+3) s} .
$$

Thus we have

$$
E_{u}=\left\{w \in \mathbb{B}_{n}: 1-|w|^{2} \in\left(2^{-2(k+3) s}, 2^{-2(k+1) s}\right), d(u, w)<s 2^{-k s}, 1-\left|\varphi_{u}(w)\right|^{2}>2^{-5 s}\right\} .
$$

The proof of (1) for $E_{v}^{\prime}$ and $F_{u}$ is similar.

The proof of (2) is a straightforward application of Lemma 2.11 to the estimate of $1-\left|\varphi_{u}(w)\right|^{2}$ above.

To prove (3), suppose $w \in \mathbb{B}_{n}$ and $\beta\left(w, E_{u}\right)<\frac{s}{4} \log 2$. Choose $z \in E_{u}$ such that $\beta(w, z)<$ $\beta\left(w, E_{u}\right)+\log 2<\frac{s}{4} \log 2+\log 2$. Then by Lemma 2.11,

$$
1-\left|\varphi_{w}(z)\right|^{2} \geq e^{-2 \beta(z, w)}>e^{-\frac{s}{2} \log 2-2 \log 2}=2^{-\frac{s}{2}-2} .
$$

By Lemma 2.12 (2) and part (1) proved above,

$$
1-|w|^{2}>\frac{1}{4}\left(1-|z|^{2}\right)\left(1-\left|\varphi_{w}(z)\right|^{2}\right)>\frac{1}{4} \cdot 2^{-2(k+3) s} \cdot 2^{-\frac{s}{2}-2}>2^{-2(k+4) s}
$$

and

$$
1-|w|^{2}<4 \frac{1-|z|^{2}}{1-\left|\varphi_{w}(z)\right|^{2}}<4 \frac{2^{-2(k+1) s}}{2^{-2-\frac{s}{2}}}<2^{-2 k s-s} .
$$

Also,

$$
d^{4}(z, w)=|1-\langle z, w\rangle|^{2}=\frac{\left(1-|z|^{2}\right)\left(1-|w|^{2}\right)}{1-\left|\varphi_{w}(z)\right|^{2}}<\frac{2^{-2(k+1) s} \cdot 2^{-2 k s-s}}{2^{-\frac{s}{2}-2}}<2^{-4 k s-2 s} .
$$

Therefore $d(z, w)<2^{-k s-\frac{s}{2}}$. Hence

$$
d(u, w) \leq d(u, z)+d(z, w)<s 2^{-k s}+2^{-k s-\frac{s}{2}}<\sqrt{2} s 2^{-k s} .
$$

Finally, by Lemma 2.11,

$$
\beta(z, u)<\log 2-\frac{1}{2} \log \left(1-\left|\varphi_{u}(z)\right|^{2}\right)<\log 2-\frac{1}{2} \log 2^{-5 s}=\left(\frac{5}{2} s+1\right) \log 2 .
$$

Therefore

$$
\beta(w, u) \leq \beta(w, z)+\beta(z, u)<\frac{s}{4} \log 2+\log 2+\left(\frac{5}{2} s+1\right) \log 2<3 s \log 2
$$

and

$$
1-\left|\varphi_{u}(w)\right|^{2}>e^{-2 \cdot 3 s \log 2}=2^{-6 s} .
$$

From inequalities (5.1) (5.2) (5.3) (5.4), we have

$$
\left\{w \in \mathbb{B}_{n}: \beta\left(w, E_{u}\right)<\frac{s}{4} \log 2\right\} \subset F_{u} .
$$

This proves (3). 
Suppose $1-|z|^{2} \in\left[2^{-2(k+2) s}, 2^{-2(k+1) s}\right)$ and $d_{S}(u, z)<s 2^{-k s-1}$. By Lemma 2.15,

$$
\begin{aligned}
d^{2}(u, z) & <d_{S}^{2}(u, z)+\left(1-|z|^{2}\right)+\left(1-|u|^{2}\right) \\
& <s^{2} 2^{-2 k s-2}+2^{-2(k+1) s}+2^{-2 k s} \\
& <s^{2} 2^{-2 k s-1} .
\end{aligned}
$$

We have

$$
1-\left|\varphi_{u}(z)\right|^{2}=\frac{\left(1-|u|^{2}\right)\left(1-|z|^{2}\right)}{|1-\langle u, z\rangle|^{2}}>\frac{2^{-2 k s} 2^{-2(k+2) s}}{s^{4} 2^{-4 k s-2}}=s^{-4} 2^{-4 s+2}>2^{-5 s} .
$$

By (1), we have $z \in E_{u}$. This proves (4).

Finally, we prove $(5)$. Suppose $z \in \mathbb{B}_{n}$. Let $k$ be the positive integer such that $2^{-2(k+1) s}<$ $1-|z|^{2} \leq 2^{-2 k s}$. If $z \in F_{u}$ and $u \in \mathcal{L}_{l}$, then by $(1), 2^{-2(l+4) s}<1-|z|^{2}<2^{-2 l s-s}$. Thus $k-3 \leq l \leq k$. Since $z \in F_{u}$ and by Lemma $2.15(2), d_{S}(z, u)<\sqrt{2} d(z, u)<2 s 2^{-l s}$. By our construction, if $u_{1} \neq u_{2}, u_{1}, u_{2} \in \mathcal{L}_{l}$, then $d_{S}\left(u_{1}, u_{2}\right)>s 2^{-s l-2}$. By Lemma 2.14, the number of $u \in \mathcal{L}_{l}$ such that $z \in F_{u}$ does not exceed $C\left(\frac{2 s 2^{-l s}+\frac{s}{2} 2^{-s l-2}}{\frac{s}{2} 2^{-s l-2}}\right)^{2 n}<C 2^{10 n}$, where $C>0$ is a constant. Thus the number of $u \in \mathcal{L}$ such that $z \in F_{u}$ does not exceed $C 2^{10 n+2}$. Replacing $s$ with $s^{\prime}$ in the proof of (3) will give us that the set $\left\{w \in \mathbb{B}_{n}: \beta\left(w, E_{v}^{\prime}\right)<\frac{s^{\prime}}{4} \log 2\right\}$ is contained in

$$
\left\{w \in \mathbb{B}_{n}: 1-|w|^{2} \in\left(2^{-2(k+4) s^{\prime}}, 2^{-2 k s^{\prime}-s^{\prime}}\right), d(v, w)<\sqrt{2} s^{\prime} 2^{-k s^{\prime}}, 1-\left|\varphi_{v}(w)\right|^{2}>2^{-6 s^{\prime}}\right\} .
$$

Thus $E_{v 3}^{\prime}$ is contained in the right hand side of the above. Thus similar proof will show that $z$ belongs to at most $C 2^{10 n}$ of the sets $F_{v}^{\prime}=E_{v 3}^{\prime}$. Altogether, the total number of $u \in \mathcal{L}$ and $v \in \mathcal{L}^{\prime}$ such that $z \in F_{u}$ or $z \in F_{v}^{\prime}$ does not exceed $M:=C 2^{10 n+3}$. This completes the proof.

Lemma 5.2. For $k$ large enough and $z \in \mathcal{Z}$ with $1-|z|^{2} \in\left[2^{-2(k+3) s}, 2^{-2 k s}\right]$, there exists $u \in \mathcal{L}_{k}$ such that

$$
d_{S}(u, z)<\frac{s}{3} 2^{-s k}
$$

Proof. By assumption, $Z$ intersects $\partial \mathbb{B}_{n}$ transversely and has no singular points on $\partial \mathbb{B}_{n}$. It is easy to see that for $z \in Z$ close enough to $\partial \mathbb{B}_{n}$, the orthogonal projection onto $N T_{z} Z$, when restricted to $Z$, is a one-sheeted analytic cover in an Euclidean neighborhood $U$ of $z$. Consider such a specific $z$. Choose an orthonormal basis $\left\{e_{1}, \cdots, e_{n}\right\}$ of $\mathbb{C}^{n}$ such that $e_{1}=\frac{z}{|z|}$ and $N T_{z} Z=\operatorname{span}\left\{e_{1}, \cdots, e_{d}\right\}$, where $d$ is the dimension of $Z$ at $z$. There is a vector-valued holomorphic function $a$ such that $\zeta \in Z \cap U$ if and only if $\zeta=\left(\zeta^{\prime}, a\left(\zeta^{\prime}\right)\right)$ under the new basis, where $\zeta^{\prime}=\left(\zeta_{1}, \cdots, \zeta_{d}\right)$, under the new basis.

We have $\frac{z^{\prime}}{|z|}=(1,0, \cdots, 0) \in \mathbb{C}^{d}$ and $a\left(z^{\prime}\right)=0$ under the new basis. Consider the paths

$$
\begin{gathered}
\gamma:[0,1] \rightarrow \mathbb{C}^{d}, t \mapsto(1-t) z^{\prime}+t \cdot r_{k-1} \frac{z^{\prime}}{|z|}, \\
\Gamma(t)=(\gamma(t), 0) \subseteq N T_{z} Z
\end{gathered}
$$

and

$$
\Lambda(t)=(\gamma(t), a(\gamma(t))) \subseteq \mathcal{Z}
$$

Note that $\Lambda(0)=\Gamma(0)=z$, so $1-|\Lambda(0)|^{2} \leq 2^{-2 s k}$. On the other hand, by the standard inverse function theorem, there is a constant $C>0$ such that for $z \in \mathcal{Z}$ close enough to $\partial \mathbb{B}_{n}$ and $\lambda^{\prime}, w^{\prime}$ 
in a sufficiently small Euclidean neighborhood of $z^{\prime}$, we have

$$
\left|a\left(\lambda^{\prime}\right)-a\left(w^{\prime}\right)\right| \leq C\left|\lambda^{\prime}-w^{\prime}\right|
$$

So when $k$ is large enough,

$$
\begin{aligned}
1-|\Lambda(1)|^{2} & =1-|\gamma(1)|^{2}-|a(\gamma(1))|^{2} \\
& \geq 1-|\gamma(1)|^{2}-C^{2}|\gamma(1)-\gamma(0)|^{2} \\
& \geq 2^{-2 s(k-1)}-C^{2} 2^{-4 s k} \\
& \geq 2^{-2 s k}
\end{aligned}
$$

By the intermediate value theorem, there exists $t_{0} \in[0,1]$ such that

$$
1-\left|\Lambda\left(t_{0}\right)\right|^{2}=2^{-2 s k}
$$

Denote $z_{0}=\Lambda\left(t_{0}\right)$. Then $z_{0} \in \mathcal{Z}_{k}$. From our construction of $\mathcal{L}_{k}$, there exists a $u \in \mathcal{L}_{k}$ such that

Also, since $\frac{z}{|z|}=\frac{\Gamma\left(t_{0}\right)}{\left|\Gamma\left(t_{0}\right)\right|}$,

$$
d_{S}\left(u, z_{0}\right) \leq s 2^{-s k-2}
$$

$$
\begin{aligned}
d_{S}^{2}\left(z, z_{0}\right) & =d_{S}^{2}\left(\Gamma\left(t_{0}\right), \Lambda\left(t_{0}\right)\right) \\
& <2 d^{2}\left(\Gamma\left(t_{0}\right), \Lambda\left(t_{0}\right)\right) \\
& =2\left(1-\left|\gamma\left(t_{0}\right)\right|^{2}\right) \\
& \leq 2\left(1-\left|\Lambda\left(t_{0}\right)\right|^{2}\right)+2\left|a\left(\gamma\left(t_{0}\right)\right)\right|^{2} \\
& \leq 2 \cdot 2^{-2 s k}+2 C^{2} 2^{-4 s k} \\
& \leq 2^{-2 s k+2} .
\end{aligned}
$$

Thus

$$
d_{S}\left(z, z_{0}\right)<2^{-s k+1}
$$

So

$$
d_{S}(z, u) \leq d_{S}\left(z, z_{0}\right)+d_{S}\left(z_{0}, u\right)<2^{-s k+1}+s 2^{-s k-2}<\frac{s}{3} 2^{-s k} .
$$

This completes the proof.

Lemma 5.3. For $s$ and $K$ large enough,

$$
\left\{w \in \mathbb{B}_{n}: 1-|w|^{2}<2^{-2 s(K+1)}, \beta(w, \mathcal{Z})<(s-1) \log 2\right\} \subset \bigcup_{k=K}^{\infty} \bigcup_{u \in \mathcal{L}_{k}} E_{u} .
$$

Proof. Suppose $w \notin \bigcup_{l=K}^{\infty} \bigcup_{u \in \mathcal{L}_{l}} E_{u}$, and $1-|w|^{2} \in\left[2^{-2 s(k+2)}, 2^{-2 s(k+1)}\right)$ for some $k \geq K$. For any $z \in \mathcal{Z}$, we want to show that $\beta(z, w) \geq(s-1) \log 2$.

If $1-|z|^{2} \notin\left(2^{-2 s(k+3)}, 2^{-2 s k}\right)$, then

$$
1-\left|\varphi_{z}(w)\right|^{2}<\min \left\{4 \frac{1-|z|^{2}}{1-|w|^{2}}, 4 \frac{1-|w|^{2}}{1-|z|}\right\}<2^{-2 s+2}
$$

Therefore

$$
\beta(z, w)>-\frac{1}{2} \log \left(1-\left|\varphi_{z}(w)\right|^{2}\right)>(s-1) \log 2 .
$$

If $1-|z|^{2} \in\left(2^{-2 s(k+3)}, 2^{-2 s k}\right)$, by Lemma 5.2, there exists $u \in \mathcal{L}_{k}$ such that

$$
d_{S}(u, z)<\frac{s}{3} 2^{-s k}
$$


Since $w \notin E_{u}$, by Lemma 5.1 (4),

$$
d_{S}(w, u) \geq s 2^{-s k-1}
$$

So

Hence

$$
d_{S}(w, z) \geq d_{S}(w, u)-d_{S}(z, u)>s 2^{-s k-1}-\frac{s}{3} 2^{-s k}=\frac{s}{6} 2^{-s k}
$$

Therefore we have

$$
d^{2}(w, z)>\frac{1}{2} d_{S}^{2}(w, z)>\frac{s^{2}}{100} 2^{-2 s k}
$$

$$
1-\left|\varphi_{z}(w)\right|^{2}=\frac{\left(1-|z|^{2}\right)\left(1-|w|^{2}\right)}{|1-\langle z, w\rangle|^{2}}<10^{4} s^{-4} 2^{-2 s}<2^{-2 s}
$$

So

$$
\beta(z, w)>-\frac{1}{2} \log \left(1-\left|\varphi_{z}(w)\right|^{2}\right)>s \log 2 .
$$

This completes the proof.

Lemma 5.3 shows that, close to the boundary, the sets $E_{u}$ cover a Bergman neighborhood of the variety $\mathcal{Z}$. In fact, we have the following lemma.

Lemma 5.4. For $s^{\prime}$ and $K$ large enough, the following are true.

(1) For any $k \geq 10 K$ and $v \in \mathcal{L}_{k}^{\prime}$,

$$
\left\{w \in \mathbb{B}_{n}: \beta\left(w, E_{v}^{\prime}\right)<s^{\prime} \log 2\right\} \cap \mathcal{Z}=\emptyset .
$$

$$
\left(\bigcup_{k=10 K}^{\infty} \bigcup_{v \in \mathcal{L}_{k}^{\prime}} E_{v}^{\prime}\right) \bigcup\left(\bigcup_{k=K}^{\infty} \bigcup_{u \in \mathcal{L}_{k}} E_{u}\right) \supset \mathbb{B}_{\delta}
$$

where $1-\delta^{2}=2^{-2 s(K+1)}$.

Proof. For $k \geq 10 K$ and $v \in \mathcal{L}_{k}^{\prime}$, by Lemma $5.1(2)$,

$$
\left\{w \in \mathbb{B}_{n}: \beta\left(w, E_{v}^{\prime}\right)<s^{\prime} \log 2\right\} \subset D\left(v, 4 s^{\prime} \log 2\right) .
$$

If $\left\{w \in \mathbb{B}_{n}: \beta\left(w, E_{v}^{\prime}\right)<s^{\prime} \log 2\right\} \cap \mathcal{Z} \neq \emptyset$, then $\beta(v, \mathcal{Z})<4 s^{\prime} \log 2$, which contradicts the definition of $\mathcal{L}_{k}^{\prime}$. This proves (1).

Suppose $w \in \mathbb{B}_{\delta}$. Then $1-|w|^{2}<2^{-2 s(K+1)}$. If $w \notin \bigcup_{l=K}^{\infty} \bigcup_{u \in \mathcal{L}_{l}} E_{u}$, then by Lemma 5.3,

$$
\beta(w, \mathcal{Z}) \geq(s-1) \log 2>9 s^{\prime} \log 2 .
$$

Suppose $1-|w|^{2} \in\left[2^{-2 s^{\prime}(k+2)}, 2^{-2 s^{\prime}(k+1)}\right)$. Then $k>10 K$. Let $w_{0}=\frac{r_{k}^{\prime}}{|w|} w$. Then

$$
1-\left|\varphi_{w}\left(w_{0}\right)\right|^{2}=\frac{\left(1-|w|^{2}\right)\left(1-\left|w_{0}\right|^{2}\right)}{\left|1-\left\langle w, w_{0}\right\rangle\right|^{2}}>\frac{1}{4} \frac{1-|w|^{2}}{1-\left|w_{0}\right|^{2}} \geq \frac{2^{-2 s^{\prime}(k+2)}}{4 \cdot 2^{-2 s^{\prime} k}}=2^{-4 s^{\prime}-2} .
$$

By Lemma 2.11, $\beta\left(w, w_{0}\right)<\log 2-\frac{1}{2} \log 2^{-4 s^{\prime}-2}<3 s^{\prime} \log 2$. Therefore

$$
\beta\left(w_{0}, z\right) \geq \beta(w, z)-\beta\left(w, w_{0}\right)>6 s^{\prime} \log 2 .
$$

Thus $w_{0} \in \mathcal{O} \cap r_{k}^{\prime} S$. By construction, there exists $v \in \mathcal{L}_{k}^{\prime}$ such that $d_{S}\left(w_{0}, v\right)<s^{\prime} 2^{-s^{\prime} k-2}$. Therefore $d_{S}(w, v)=d_{S}\left(w_{0}, v\right)<s^{\prime} 2^{-s^{\prime} k-2}$. By Lemma 5.1 (4), $w \in E_{v}^{\prime}$. This completes the proof. 


\section{Local Decomposition Formulas}

To show that the $I$ in Theorem 4.1 satisfies Hypothesis 1, we need to construct decompositions $\sum p_{i j} f_{i j}$ on the sets $E_{u 3}$ (or $\left.E_{v 3}^{\prime}\right)$, for $f \in I$. It is more convenient to construct the decompositions on their images under a Möbius transform.

Lemma 6.1. There exist a positive integer $N$ and a constant $C>0$, depending on $I$, such that the following hold. For any $R>0$ sufficiently large, let $\left\{E_{u}\right\}_{u \in \mathcal{L}},\left\{F_{u}\right\}_{u \in \mathcal{L}},\left\{E_{v}^{\prime}\right\}_{v \in \mathcal{L}^{\prime}}$ and $\left\{F_{v}^{\prime}\right\}_{v \in \mathcal{L}^{\prime}}$ be as in the beginning of Section 5. Then there exists a positive integer $K$ such that the following hold.

(1) For each $u \in \mathcal{L}_{k}, k \geq K$, there is a finite set of polynomials $\left\{p_{l}\right\} \subset I$, $\sup _{l} \operatorname{deg} p_{l} \leq N$, and linear maps $I \rightarrow \operatorname{Hol}\left(E_{u 3}\right), f \mapsto f_{l}$ satisfying the following inequalities.

(i) $\int_{E_{u 3}}\left|\sum_{l} p_{l} f_{l}-f\right|^{2} d v \leq C \epsilon_{R}^{2} \int_{F_{u}}|f|^{2} d v$.

(ii) $\int_{E_{u 3}}\left(\sum_{l}\left|p_{l} f_{l}\right|\right)^{2} d v \leq C \int_{F_{u}}|f|^{2} d v$.

Here $\epsilon_{R}=\epsilon_{R, 0}$ is defined in Lemma 2.20.

(2) For each $v \in \mathcal{L}_{k}^{\prime}, k \geq 10 K$, there is a $p \in I$ such that $p$ is non-vanishing on $F_{v}^{\prime}$ and $\operatorname{deg} p \leq N$.

Some preparations are needed for the proof of Lemma 6.1.

Lemma 6.2. For any positive integer $m, k$, and $x, y \in \mathbb{C}, x, y \neq 0$, there are constants $\left\{c_{j}\right\}_{j=0}^{m-1},\left\{d_{j}\right\}_{j=0}^{k-1}$, such that

$$
\frac{1}{x^{k}}=\sum_{j=0}^{m-1} c_{j} \frac{(x-y)^{j}}{y^{k+j}}+\sum_{j=0}^{k-1} d_{j} \frac{(x-y)^{m}}{x^{k-j} y^{m+j}}
$$

Proof. We prove by induction on $m$. For $m=1$ and any $k$,

$$
\frac{1}{x^{k}}=\frac{1}{y^{k}}+\left(\frac{1}{x^{k}}-\frac{1}{y^{k}}\right)=\frac{1}{y^{k}}-\sum_{j=0}^{k-1} \frac{x^{j} y^{k-1-j}}{x^{k} y^{k}}(x-y)=\frac{1}{y^{k}}-\sum_{j=0}^{k-1} \frac{(x-y)}{x^{k-j} y^{1+j}} .
$$

Suppose the equation holds for $m-1$ and any $k$. Then

$$
\begin{aligned}
\frac{1}{x^{k}} & =\sum_{j=0}^{m-2} c_{j}^{\prime} \frac{(x-y)^{j}}{y^{k+j}}+\sum_{j=0}^{k-1} d_{j}^{\prime} \frac{(x-y)^{m-1}}{x^{k-j} y^{m-1+j}} \\
& =\sum_{j=0}^{m-2} c_{j}^{\prime} \frac{(x-y)^{j}}{y^{k+j}}+\sum_{j=0}^{k-1} d_{j}^{\prime} \frac{(x-y)^{m-1}}{y^{m-1+j}}\left(\frac{1}{y^{k-j}}-\sum_{i=0}^{k-j-1} \frac{(x-y)}{x^{k-j-i} y^{1+i}}\right) \\
& =\sum_{j=0}^{m-1} c_{j} \frac{(x-y)^{j}}{y^{k+j}}+\sum_{l=0}^{k-1} d_{l} \frac{(x-y)^{m}}{x^{k-l} y^{m+l}} .
\end{aligned}
$$

This completes the proof.

The following lemma is elementary.

Lemma 6.3. Suppose $0<a<b$ and $d$ is a positive integer. Write $B_{a}^{b}=\left\{z \in \mathbb{C}^{d}: a<|z|<b\right\}$. Then for any $f \in \operatorname{Hol}\left(b \mathbb{B}_{d}\right)$ we have 
(1)

$$
\int_{b \mathbb{B}_{d}}|f|^{2} d v \leq \frac{b}{b-a} \int_{B_{a}^{b}}|f|^{2} d v
$$

(2) For any multi-index $\alpha=\left(\alpha_{1}, \cdots, \alpha_{d}\right)$,

$$
\int_{B_{a}^{b}} f(w) \overline{w^{\alpha}} d v(w)=c_{a, b} \partial^{\alpha} f(0)
$$

Here $c_{a, b, \alpha}=\frac{1}{\alpha !} \int_{B_{a}^{b}}\left|w^{\alpha}\right|^{2} d v(w)$.

Lemma 6.4. Let $d, m$ be positive integers, $0<d<n$. For $R>0$ and a positive integer $k$ that are large enough, the following hold. Let $s=10 s^{\prime}$, where $s^{\prime}$ is the unique integer satisfying $\left(s^{\prime}-1\right) \log 2 \leq 16 R<s^{\prime} \log 2$. Write $r=\sqrt{1-2^{-2 k s}}, u=(r, 0, \cdots, 0)$, and define $E:=\tilde{E}_{u}$, $F:=\tilde{F}_{u}$ as in the beginning of Section 5 , i.e.,

$$
E=\left\{z \in \mathbb{B}_{n}: 1-|z|^{2}>2^{-5 s},|1-\langle z, u\rangle|>s^{-2}, \frac{1-|z|^{2}}{|1-\langle z, u\rangle|^{2}}<2^{-2 s}\right\},
$$

and

$$
F=\left\{z \in \mathbb{B}_{n}: 1-|z|^{2}>2^{-6 s},|1-\langle z, u\rangle|>\frac{1}{2} s^{-2}, \frac{1-|z|^{2}}{|1-\langle z, u\rangle|^{2}}<2^{-s}\right\} .
$$

Write $U^{\prime}=\pi(F)$, where $\pi$ is the projection $\pi: \mathbb{C}^{n} \rightarrow \mathbb{C}^{d}, z \mapsto z^{\prime}:=\left(z_{1}, \cdots, z_{d}\right)$. Then there exist $\delta>0$, depending only on $d, m, R$, and $C>0$, depending only on $d, m$, with the following properties.

Suppose $a: U^{\prime} \rightarrow \mathbb{C}^{n-d}$ is holomorphic and $\left|a\left(z^{\prime}\right)\right|<\delta, \forall z^{\prime} \in U^{\prime}$. Write $A=\left\{\left(z^{\prime}, a\left(z^{\prime}\right)\right)\right.$ : $\left.z^{\prime} \in U^{\prime}\right\}$. Let $\left\{P_{\alpha}=P_{\left.\pi\right|_{m A}, \alpha}\right\}$ be the set of canonical defining functions with respect to $\left.\pi\right|_{m A}$. Here $\alpha=\left(\alpha_{d+1}, \cdots, \alpha_{n}\right),|\alpha|=m$. Then there exist linear maps $f \mapsto f_{\alpha}$, where $f \in \operatorname{Hol}\left(\mathbb{B}_{n}\right)$, $\left.f\right|_{m A}=0$, and $f_{\alpha} \in \operatorname{Hol}\left(E_{3}\right)$, with the following properties.

$$
\int_{E_{3}}\left|f-\sum_{|\alpha|=m} P_{\alpha} f_{\alpha}\right|^{2} d v \leq C \epsilon_{R}^{2} \int_{F}|f|^{2} d v .
$$

(2) For any $\alpha, \beta,|\alpha|=|\beta|=m$,

$$
\int_{E_{3}}\left|P_{\beta} f_{\alpha}\right|^{2} d v \leq C \int_{F}|f|^{2} d v
$$

Here $\epsilon_{R}=\epsilon_{R, 0}$ is the constant defined in Lemma 2.20.

Proof. To simplify notations, we write $\pi(z)=\left(z^{\prime}, 0\right)$ and $p(z)=\left(z^{\prime}, a\left(z^{\prime}\right)\right)$.

We will construct the functions $f_{\alpha}$ by decomposing the reproducing kernel $K_{w}(z)$. For $z \in F$ and $w \in \mathbb{B}_{n}$, applying Lemma 6.2 for $x=1-\langle z, w\rangle, y=1-\langle p(z), w\rangle$, we get

$$
\begin{aligned}
K_{w}(z) & =\sum_{j=0}^{m-1} c_{j} \frac{\left\langle a\left(z^{\prime}\right)-z^{\prime \prime}, w^{\prime \prime}\right\rangle^{j}}{(1-\langle p(z), w\rangle)^{n+1+j}}+\sum_{j=0}^{n} d_{j} \frac{\left\langle a\left(z^{\prime}\right)-z^{\prime \prime}, w^{\prime \prime}\right\rangle^{m}}{(1-\langle z, w\rangle)^{n+1-j}(1-\langle p(z), w\rangle)^{m+j}} \\
& =I(z, w)+I I(z, w) .
\end{aligned}
$$

Notice that $\left\langle a\left(z^{\prime}\right)-z^{\prime \prime}, w^{\prime \prime}\right\rangle^{m}=P_{\left.\pi\right|_{m A}}\left(z, w^{\prime \prime}\right)=\sum_{|\alpha|=m} P_{\alpha}(z) \overline{w^{\prime \prime \alpha}}$. Write

$$
G(z, w)=\sum_{j=0}^{n} d_{j} \frac{1}{(1-\langle z, w\rangle)^{n+1-j}(1-\langle p(z), w\rangle)^{m+j}}
$$


Then $I I(z, w)=\sum_{|\alpha|=m} G(z, w) \overline{w^{\prime \prime \alpha}} P_{\alpha}(z)$. Let

$$
f_{\alpha}(z)=c_{R}^{-1} \int_{F_{1}} f(w) G(z, w) \overline{w^{\prime \prime \alpha}} d v(w) .
$$

Here $c_{R}=c_{R, 0}$ is the constant in Lemma 2.20, Then

$$
\left(P_{\beta} f_{\alpha}\right)(z)=c_{R}^{-1} \int_{F_{1}} f(w) G(z, w) \overline{w^{\prime \prime \alpha}} P_{\beta}(z) d v(w) .
$$

We will show that the functions satisfy inequalities (1) and (2).

First, we prove inequality (2). Notice that by definition, $1-|z|^{2}>2^{-6 s}, \forall z \in F$. If $\delta<2^{-6 s-2}$ and $\left|a\left(\xi^{\prime}\right)\right| \leq \delta, \forall \xi^{\prime} \in U^{\prime}$, then for any $z \in E_{3}, w \in F$, we have

$|1-\langle p(z), w\rangle|=\left|1-\left\langle z^{\prime}, w^{\prime}\right\rangle-\left\langle a\left(z^{\prime}\right), w^{\prime \prime}\right\rangle\right| \geq\left|1-\left\langle z^{\prime}, w^{\prime}\right\rangle\right|-\left|a\left(z^{\prime}\right)\right|>\left|1-\left\langle z^{\prime}, w^{\prime}\right\rangle\right|-2^{-6 s-2} \geq \frac{1}{2}\left|1-\left\langle z^{\prime}, w^{\prime}\right\rangle\right|$.

Here the last inequality holds because

$$
\left|1-\left\langle z^{\prime}, w^{\prime}\right\rangle\right| \geq 1-|w| \geq \frac{1}{2}\left(1-|w|^{2}\right)>2^{-6 s-1} .
$$

Thus

$$
|G(z, w)| \lesssim \sum_{j=0}^{n} \frac{1}{|1-\langle z, w\rangle|^{n+1-j}\left|1-\left\langle z^{\prime}, w^{\prime}\right\rangle\right|^{m+j}} .
$$

For each pair $\alpha, \beta,|\alpha|=|\beta|=m$ and $z \in E_{3}, w \in F$, since

$$
\left|\overline{w^{\prime \prime \alpha}}\right| \leq|w|^{m / 2} \leq\left(1-\left|w^{\prime}\right|^{2}\right)^{m / 2} \lesssim\left|1-\left\langle z^{\prime}, w^{\prime}\right\rangle\right|^{m / 2}
$$

and

$$
\left|P_{\beta}(z)\right| \leq\left|a\left(z^{\prime}\right)-z^{\prime \prime}\right|^{m / 2} \lesssim\left|1-\left\langle z^{\prime}, w^{\prime}\right\rangle\right|^{m / 2}
$$

we have

$$
\left|G(z, w) \overline{w^{\prime \prime \alpha}} P_{\beta}(z)\right| \lesssim \sum_{j=0}^{n} \frac{1}{|1-\langle z, w\rangle|^{n+1-j}\left|1-\left\langle z^{\prime}, w^{\prime}\right\rangle\right|^{j}} \lesssim \frac{1}{|1-\langle z, w\rangle|^{n+1}}+\frac{1}{\left|1-\left\langle z^{\prime}, w^{\prime}\right\rangle\right|^{n+1}} .
$$

By (6.1) and Lemma 2.20, we obtain inequality (2).

Next, we prove (1). For any $z \in E_{3}$, by Lemma $5.1(2),(3), D(z, R) \subset F$. By Lemma 2.20 $(3)$,

$$
\int_{F} f(w) K_{w}(z) d v(w)-c_{R} f(z)=\int_{F \backslash D(z, R)} f(w) K_{w}(z) d v(w) .
$$

Thus

$$
\begin{aligned}
& f(z)-\sum_{\alpha} P_{\alpha} f_{\alpha} \\
= & c_{R}^{-1}\left(\int_{F} f(w) K_{w}(z) d v(w)-\int_{F \backslash D(z, R)} f(w) K_{w}(z) d v(w)-\int_{F} f(w) I I(z, w) d v(w)\right) \\
= & c_{R}^{-1}\left(\int_{F} f(w) I(z, w) d v(w)-\int_{F \backslash D(z, R)} f(w) K_{w}(z) d v(w)\right) .
\end{aligned}
$$

Each term of $I$ has the form $\frac{\left(a\left(z^{\prime}\right)-z^{\prime \prime}\right)^{\alpha} \overline{w^{\prime \prime \alpha}}}{(1-\langle p(z), w\rangle)^{n+1+j}}$, where $0 \leq j \leq m-1$ and $|\alpha|=j$. We want to prove that their integrals with $f$ are small. First, since $E_{3} \subset F, E_{3} \subset\left\{z \in \mathbb{B}_{n}: 1-|z|^{2}>2^{-6 s}\right\}$, 
which is contained in a compact subset of $\mathbb{B}_{n}$, we can find $C_{1}>0$, depending only on $m, R$, such that

$$
\left|\frac{\left(a\left(z^{\prime}\right)-z^{\prime \prime}\right)^{\alpha} \overline{w^{\prime \prime \alpha}}}{(1-\langle p(z), w\rangle)^{n+1+j}}-\frac{\left(-z^{\prime \prime}\right)^{\alpha} \overline{w^{\prime \prime \alpha}}}{(1-\langle\pi(z), w\rangle)^{n+1+j}}\right| \leq C_{1}\left|a\left(z^{\prime}\right)\right| \leq C_{1} \delta, \quad \forall z \in E_{3}, w \in F .
$$

Therefore

$$
\left|\int_{F} f(w) I(z, w) d v(w)-\int_{F} f(w) \sum_{j=0}^{m-1} c_{j} \frac{\left\langle-z^{\prime \prime}, w^{\prime \prime}\right\rangle^{j}}{\left(1-\left\langle z^{\prime}, w^{\prime}\right\rangle\right)^{n+1+j}} d v(w)\right| \lesssim C_{1} \delta \int_{F}|f(w)| d v(w) .
$$

Notice that for each $w^{\prime} \in U^{\prime}$, we have $\left|1-\left\langle w^{\prime}, u^{\prime}\right\rangle\right|>\frac{1}{2} s^{-2}$, and therefore

$$
\pi^{-1}\left(w^{\prime}\right) \cap F=\left\{\left(w^{\prime}, w^{\prime \prime}\right): 1-\left|w^{\prime}\right|^{2}-2^{-s}\left|1-\left\langle w^{\prime}, u^{\prime}\right\rangle\right|^{2}<\left|w^{\prime \prime}\right|^{2}<1-\left|w^{\prime}\right|^{2}-2^{-6 s}\right\} .
$$

Each fiber is either a spherical shell or a ball in $\mathbb{C}^{n-d}$. From this and Lemma 6.3 (2), it is easy to see that for $z^{\prime} \in \pi\left(E_{3}\right)$,

$$
\begin{aligned}
& \left|\int_{F} f(w) \sum_{j=0}^{m-1} c_{j} \frac{\left\langle-z^{\prime \prime}, w^{\prime \prime}\right\rangle^{j}}{\left(1-\left\langle z^{\prime}, w^{\prime}\right\rangle\right)^{n+1+j}} d v(w)\right| \\
\lesssim & \sum_{j=0}^{m-1} \int_{U^{\prime}} \frac{1}{\left|1-\left\langle z^{\prime}, w^{\prime}\right\rangle\right|^{n+1+j}} d v\left(w^{\prime}\right) \sup _{z^{\prime} \in \pi\left(E_{3}\right),|\alpha| \leq m-1}\left|\partial^{\alpha} f\left(z^{\prime}, 0\right)\right| \\
\lesssim & 2^{6 s(n+m)} \sup _{z^{\prime} \in \pi\left(E_{3}\right),|\alpha| \leq m-1}\left|\partial^{\alpha} f\left(z^{\prime}, 0\right)\right| .
\end{aligned}
$$

It remains to show that $\sup _{z^{\prime} \in \pi\left(E_{3}\right),|\alpha| \leq m-1}\left|\partial^{\alpha} f\left(z^{\prime}, 0\right)\right|$ is sufficiently small. Obtain $F_{1}$ by filling the "holes" in $F$, i.e.,

$$
F_{1}=\left\{\left(w^{\prime}, w^{\prime \prime}\right) \in \mathbb{B}_{n}: w^{\prime} \in U^{\prime},\left|w^{\prime \prime}\right|^{2}<1-\left|w^{\prime}\right|^{2}-2^{-6 s}\right\} .
$$

By Lemma 5.1, $E_{3} \subset D(0,4 s \log 2)$ and $F \supset E_{4}$. Thus for $\delta$ small enough, the set of points $\left\{\pi(z): z \in E_{3}\right\} \cup\left\{p(z): z \in E_{3}\right\}$ has a positive Euclidean distance (independent of $k$ ) to the boundary of $F_{1}$. So there exists a constant $C_{2}>0$ (depending only on $d, m, R$ ) such that $\forall z \in E_{3}, \forall g \in \operatorname{Hol}\left(F_{1}\right)$ and $\forall \alpha,|\alpha| \leq m-1$,

$$
\left|\left(\partial^{\alpha} g\right)(p(z))-\left(\partial^{\alpha} g\right)(\pi(z))\right|^{2} \leq C_{2}^{2}|p(z)-\pi(z)|^{2} \int_{F_{1}}|g(w)|^{2} d v(w) \leq C_{2}^{2} \delta^{2} \int_{F_{1}}|g(w)|^{2} d v(w) .
$$

Since $\left.f\right|_{m A}=0$, we have $\left(\partial^{\alpha} f\right)(p(z))=0$ for all $z \in E_{3}$ and $\alpha=\left(\alpha_{d+1}, \cdots, \alpha_{n}\right),|\alpha| \leq m-1$. Thus

$$
\sup _{z^{\prime} \in \pi\left(E_{3}\right),|\alpha| \leq m-1}\left|\left(\partial^{\alpha} f\right)\left(z^{\prime}, 0\right)\right|^{2} \leq C_{2}^{2} \delta^{2} \int_{F_{1}}|f(w)|^{2} d v(w), \quad \forall z \in E_{3} .
$$

We need to compare the $L^{2}$-norms on $F_{1}$ and $F$. For any $w^{\prime} \in U^{\prime}$, if $\left.1-\left|w^{\prime}\right|^{2}-2^{-s}\left|1-\left\langle w^{\prime}, u^{\prime}\right\rangle\right|^{2}\right\rangle$ 0 , then for $s$ large enough, we have

$$
\begin{aligned}
& \frac{1-\left|w^{\prime}\right|^{2}-2^{-6 s}}{\left(1-\left|w^{\prime}\right|^{2}-2^{-6 s}\right)-\left(1-\left|w^{\prime}\right|^{2}-2^{-2 s}\left|1-\left\langle w^{\prime}, u^{\prime}\right\rangle\right|^{2}\right)} \\
= & \frac{1-\left|w^{\prime}\right|^{2}-2^{-6 s}}{2^{-2 s}\left|1-\left\langle w^{\prime}, u^{\prime}\right\rangle\right|^{2}-2^{-6 s}} \\
\leq & \frac{1}{2^{-2 s-2} s^{-4}-2^{-6 s}} \leq 2^{2 s+3} s^{4} .
\end{aligned}
$$


Then by Lemma 6.3 and a simple double integral argument, we have

$$
\int_{F_{1}}|g|^{2} d v \leq 2^{2 s+3} s^{4} \int_{F}|g|^{2} d v, \quad \forall g \in \operatorname{Hol}\left(F_{1}\right) .
$$

Combining inequalities (6.5) (6.8) (6.9), we get

$$
\left|\int_{F} f(w) \sum_{j=0}^{m-1} c_{j} \frac{\left\langle-z^{\prime \prime}, w^{\prime \prime}\right\rangle^{j}}{\left(1-\left\langle z^{\prime}, w^{\prime}\right\rangle\right)^{n+1+j}} d v(w)\right| \lesssim 2^{6 s(n+m)} C_{2} \delta \cdot 2^{s+2} s^{2}\left(\int_{F}|f|^{2} d v\right)^{1 / 2} .
$$

Then by inequality (6.4) and Holder's inequality, we get

$$
\left|\int_{F} f(w) I(z, w) d v(w)\right| \lesssim\left(C_{1} \delta+2^{6 s(n+m)} C_{2} \delta \cdot 2^{s+2} s^{2}\right)\left(\int_{F}|f|^{2} d v\right)^{1 / 2} .
$$

If we choose $\delta$ small enough, we can make $\left(C_{1} \delta+2^{6 s(n+m)} C_{2} \delta \cdot 2^{s+2} s^{2}\right) \leq \epsilon_{R}$. Then inequality (1) follows from inequality (6.3), Lemma 2.20 and our estimates above. This completes the proof.

The following Lemma is a simplified version of Lemma 6.1 (1).

Lemma 6.5. Suppose $J$ is a prime ideal in $\mathbb{C}\left[z_{1}, \cdots, z_{n}\right]$. Write $A=Z(J)$. Suppose A has no singular point in $\partial \mathbb{B}_{n}$ and intersects $\partial \mathbb{B}_{n}$ transversely. Then for a positive integer $m$ there exist a positive integer $N$ and constant $C>0$ with the following property. For $R>0$ sufficiently large, there exists a positive integer $K$ such that the following hold. Let $s^{\prime}$ be the positive integer such that $\left(s^{\prime}-1\right) \log 2 \leq 16 R<s^{\prime} \log 2$ and $s=10 s^{\prime}$,

(1) For each $u \in A, 1-|u|^{2}=2^{-2 s k}, k \geq K$, define $E_{u}$ and $F_{u}$ as in the beginning of Section 5. Then there is a finite set of polynomials $\left\{p_{l}\right\} \subset J^{m}, \sup _{l} \operatorname{deg} p_{l} \leq N$, and linear maps $J \rightarrow \operatorname{Hol}\left(E_{u 3}\right), f \mapsto f_{l}$ satisfying the following inequalities.

(i) $\int_{E_{u 3}}\left|\sum_{l} p_{l} f_{l}-f\right|^{2} d v \leq C \epsilon_{R}^{2} \int_{F_{u}}|f|^{2} d v$.

(ii) $\int_{E_{u 3}}\left(\sum_{l}\left|p_{l} f_{l}\right|\right)^{2} d v \leq C \int_{F_{u}}|f|^{2} d v$.

(2) For each $v \in \mathbb{B}_{n}, \beta(v, A)>5 s^{\prime} \log 2,1-|v|^{2}=2^{-2 s^{\prime} k}, k \geq 10 K$, define $F_{v}^{\prime}$ as in the beginning of Section [5. Then there exists a polynomial $p \in J^{m}$ such that $p$ is nonvanishing on $F_{v}^{\prime}$ and $\operatorname{deg} p \leq N$.

Proof. We want to apply Lemma 6.4. A Möbius transform $\varphi_{u}$ will allow us to transfer between decompositions on $E_{u 3}$ and $\tilde{E}_{u 3}$. Also, we need to make adjustments so that the generating functions $p_{l}$ are polynomials in $J^{m}$.

Denote $d=\operatorname{dim} A$. For any $\zeta \in \partial \mathbb{B}_{n} \cap A$, since $A$ intersects $\partial \mathbb{B}_{n}$ transversely at $\zeta, A$ is not contained in $\zeta^{\perp}+\zeta$. By Theorem 8.6, the set

$$
\tilde{\mathcal{U}}_{\zeta}=\left\{l \in U\left(\zeta^{\perp}\right): \overline{(l \oplus 1)\left(N T_{\zeta} A\right)}{ }^{\perp} \cap \overline{A-\zeta}=\emptyset\right\}
$$

is dense in $U\left(\zeta^{\perp}\right)$. Here we denote $L^{\perp}$ the orthogonal complement of a linear space $L$ in the complex projective space $\mathbb{P}_{n}$ (cf. Appendix) and $U\left(\zeta^{\perp}\right)$ the space of all unitary transforms on $\zeta^{\perp} \cong \mathbb{C}^{n-1}$. We consider $l \oplus 1$ as acting on the $\zeta^{\perp}$ by $l$ and acting on $\mathbb{C} \zeta$ as identity.

Let $\delta>0$ be the constant in Lemma 6.4 determined by $d, m, R$. If $\overline{N T_{\zeta} A} \cap \overline{A-\zeta}=\emptyset$, then take $L_{\zeta}=N T_{\zeta} A$. Otherwise, choose $l \in \tilde{\mathcal{U}}_{\zeta}$ close enough to the identity $I_{\zeta^{\perp}}$ so that the Hausdorff distance

$$
\operatorname{dist}_{H}\left(L_{\zeta} \cap \mathbb{B}_{n}, N T_{\zeta} A \cap \mathbb{B}_{n}\right) \leq \delta / 4
$$


where $L_{\zeta}=(l \oplus 1)\left(N T_{\zeta} A\right)$. In either case we have $\zeta \in L_{\zeta},{\overline{L_{\zeta}}}^{\perp} \cap \overline{A-\zeta}=\emptyset$, and

$$
\operatorname{dist}_{H}\left(L_{\zeta} \cap \mathbb{B}_{n}, N T_{\zeta} A \cap \mathbb{B}_{n}\right) \leq \delta / 4 \text {. }
$$

Choose a new basis $\left\{e_{1}, \cdots, e_{n}\right\}$ such that $L_{\zeta}=\operatorname{span}\left\{e_{1}, \cdots, e_{d}\right\}$. Denote $\pi_{\zeta}$ the projection from $\mathbb{C}^{n}$ onto $L_{\zeta}$.

By Lemma 8.4, $\left.\pi_{\zeta}\right|_{A-\zeta}$ is proper. Since $\zeta$ is a regular point of $A$ and $A$ intersects $\partial \mathbb{B}_{n}$ transversely, $\pi_{\zeta}$ defines a one-sheeted analytic cover of $A-\zeta$ in a small neighborhood of 0 . Apply Theorem 8.5 to $A-\zeta$ and $L_{\zeta}$. Let $U_{1}, \mathcal{U}$ and $\mathcal{W}$ be as in Theorem 8.5. Thus for any $w \in \mathcal{W}$ and $l \in \mathcal{U}$, the function

$$
\frac{P_{\left.\pi_{\zeta}\right|_{l(A-\zeta)}}(z, w)}{P_{\left.\pi_{\zeta}\right|_{l(A-\zeta) \cap U_{1}}}(z, w)}
$$

is non-vanishing and holomorphic in $U_{1}$.

Start with an open neighborhood $U$ of $\zeta$. For $u \in A \cap U$, let $L_{u}=L_{\zeta} \cap u^{\perp}+\mathbb{C} u$ and denote $\pi_{u}$ the projection from $\mathbb{C}^{n}$ onto it. Since $\zeta \in L_{\zeta}$, the definition is consistent at $\zeta$, and the spaces $L_{u}$ vary continuously with $u$. Thus for $u$ close enough to $\zeta$, we can find $l_{u} \in \mathcal{U}$ such that $l_{u}^{-1}\left(L_{\zeta}\right)=L_{u}$. So $\pi_{u}=l_{u}^{-1} \pi_{\zeta} l_{u}$. For any $w \in \mathcal{W}$

$$
\frac{P_{\left.\pi_{\zeta}\right|_{l_{u}(A-\zeta)}}(z, w)}{P_{\left.\pi_{\zeta}\right|_{l_{u}(A-\zeta) \cap U_{1}}}(z, w)}
$$

is non-vanishing in $U_{1}$. Equivalently, for any $w \in \mathcal{W}$,

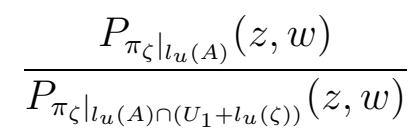

is non-vanishing in $U_{1}+l_{u}(\zeta)$. Note that the definition of canonical defining functions depend on the choice of a basis. Let $e_{u, i}=l_{u}^{-1}\left(e_{i}\right)$. By Remark2.7, under the new basis $\left\{e_{u, 1}, \cdots, e_{u, n}\right\}$, we have

$$
\psi_{w}(z):=\frac{P_{\left.\pi_{u}\right|_{A}}(z, w)}{P_{\left.\pi_{u}\right|_{A \cap\left(l_{u}^{-1} U_{1}+\zeta\right)}}(z, w)}
$$

is non-vanishing for $z \in l_{u}^{-1} U_{1}+\zeta$ and $w \in \mathcal{W}$. It is easy to see that $l_{u}^{-1}\left(U_{1}\right)+\zeta \supset U_{2}, \forall u \in U$, for some open neighborhood $U_{2}$ of $\zeta$. Let us use $\hat{P}$ with appropriate subscripts to denote the locally defined canonical defining functions depending on a one-sheeted analytic cover on the piece of manifold $A \cap U$, or its image under a Möbius transform. Then we have shown that for any $u \in U$ and $w \in \mathcal{W}$,

is non-vanishing on $U_{2}$.

$$
\psi_{w}(z)=\frac{P_{\left.\pi_{u}\right|_{A}}(z, w)}{\hat{P}_{\left.\pi_{u}\right|_{A}}(z, w)}
$$

On the other hand, both $L_{u}$ and the normal tangent spaces $N T_{u} A$ vary continuously. By shrinking the neighborhood $U$ we can also assume that

$$
\operatorname{dist}_{H}\left(L_{u} \cap \mathbb{B}_{n}, N T_{u} A \cap \mathbb{B}_{n}\right) \leq \delta / 2, \quad \forall u \in U \cap A .
$$

By Lemma 4.5 and 5.1 (2), again, by shrinking $U$, we also have for any $u \in U$,

$$
\operatorname{dist}\left(z, N T_{u} A\right) \leq \delta / 2, \quad \forall z \in \varphi_{u}(A) \cap \tilde{F}_{u} .
$$

Thus

$$
\operatorname{dist}\left(z, L_{u}\right) \leq \delta, \quad \forall z \in \varphi_{u}(A) \cap \tilde{F}_{u}
$$


For any $f \in J^{m}$, define $U_{u} f(z)=f \circ \varphi_{u}(z) \cdot k_{u}(z)$, where $k_{u}(z)=\frac{\left(1-|u|^{2}\right)^{(n+1) / 2}}{(1-\langle z, u\rangle)^{n+1}}$. Then $\left.U_{u} f\right|_{m \varphi_{u}(A)}=0$. Applying Lemma 6.4 to $F=\tilde{F}_{u}$ and $\varphi_{u}(A)$, we can find $F_{\alpha}$ such that

$$
\int_{\tilde{E}_{u 3}}\left|U_{u} f-\sum_{|\alpha|=m_{j}} \hat{P}_{\alpha} F_{\alpha}\right|^{2} d v \leq C \epsilon_{R}^{2} \int_{\tilde{F}_{u}}\left|U_{u} f\right|^{2} d v
$$

and

$$
\int_{\tilde{E}_{u 3}}\left(\sum_{|\alpha|=m_{j}}\left|\hat{P}_{\alpha} F_{\alpha}\right|\right)^{2} d v \leq C \int_{\tilde{F}_{u}}\left|U_{u} f\right|^{2} d v
$$

Here $\hat{P}_{\alpha}=\hat{P}_{\left.\pi_{u}\right|_{m \varphi_{u}(A)}, \alpha}$, i.e., $\sum_{|\alpha|=m} \hat{P}_{\alpha}(z) \overline{w^{\alpha}}=\hat{P}_{\left.\pi_{u}\right|_{\varphi_{u}(A)}}(z, w)$. Therefore

$$
\begin{aligned}
& \int_{E_{u 3}}\left|f(z)-\sum_{|\alpha|=m} \hat{P}_{\alpha} \circ \varphi_{u}(z) F_{\alpha} \circ \varphi_{u}(z) k_{u}(z)\right|^{2} d v(z) \\
= & \int_{\tilde{E}_{u 3}}\left|U_{u} f-\sum_{|\alpha|=m} \hat{P}_{\alpha} F_{\alpha}\right|^{2} d v(z) \\
\leq & C \epsilon_{R}^{2} \int_{\tilde{F}_{u}}\left|U_{u} f\right|^{2} d v(z) \\
= & C \epsilon_{R}^{2} \int_{F_{u}}|f(z)|^{2} d v(z) .
\end{aligned}
$$

Similarly, for each pair $\alpha, \beta,|\alpha|=|\beta|=m$,

$$
\int_{E_{u 3}}\left|\hat{P}_{\beta} \circ \varphi_{u}(z) F_{\alpha} \circ \varphi_{u}(z) k_{u}(z)\right|^{2} d v(z) \leq C \int_{F_{u}}|f(z)|^{2} d v(z) .
$$

Using the formula for $\varphi_{u}$, it is easy to verify that

$$
\hat{P}_{\left.\pi_{u}\right|_{\varphi u(A)}}\left(\varphi_{u}(z), w\right)=-\frac{\left(1-|u|^{2}\right)^{1 / 2}}{1-\langle z, u\rangle} \hat{P}_{\left.\pi_{u}\right|_{A}}(z, w) .
$$

Let $\Gamma=\left\{\alpha=\left(\alpha_{d+1}, \cdots, \alpha_{n}\right):|\alpha|=m\right\}$ and let $K=\# \Gamma$. By Lemma 8.2, we can choose $K$ distinct points $\left\{w_{i}\right\}_{i=1}^{K} \subset \mathcal{W}$ (independent of $u$ ) such that the vectors $\left\{\left(w_{i}^{\alpha}\right)_{\alpha \in \Gamma}\right\}_{i=1}^{K}$ form a basis of $\mathbb{C}^{K}$. Let $W$ be the $K \times K$ matrix $\left[\overline{w_{i}^{\alpha}}\right]_{\alpha \in \Gamma, i=1, \cdots, K}$. Then $W$ is invertible. Suppose $W^{-1}=\left(c_{i, \alpha}\right)$.

Denote $p_{i}(z)=P_{\left.\pi_{u}\right|_{A}}^{m}\left(z, w_{i}\right)$ and $\psi_{i}=\psi_{w_{i}}, i=1, \cdots, K$. Since $J$ is prime, by Theorem 8.3 and Hilbert's Nullstellensatz, each $p_{i}$ is a polynomial in $J^{m}$. Denote $N_{0}$ the upper bound of the degrees of the canonical defining functions of $A$, as in Theorem 8.3 . Then we have $\operatorname{deg} p_{i} \leq m N_{0}$. Set $N=m N_{0}$. Let

$$
f_{i}(z)=\sum_{\alpha}\left(-\frac{\left(1-|u|^{2}\right)^{1 / 2}}{1-\langle z, u\rangle}\right)^{m} c_{i, \alpha} \psi_{i}(z)^{-m} F_{\alpha} \circ \varphi_{u}(z) k_{u}(z)
$$


then

$$
\begin{aligned}
p_{i}(z) f_{i}(z) & =\sum_{\alpha}\left(-\frac{\left(1-|u|^{2}\right)^{1 / 2}}{1-\langle z, u\rangle}\right)^{m} c_{i, \alpha} \hat{P}_{\left.\pi_{u}\right|_{A}}\left(z, w_{i}\right)^{m} F_{\alpha} \circ \varphi_{u}(z) k_{u}(z) \\
& =\sum_{\alpha} c_{i, \alpha} \hat{P}_{\left.\pi_{u}\right|_{\varphi}(A)}^{m}\left(\varphi_{u}(z), w_{i}\right) F_{\alpha} \circ \varphi_{u}(z) k_{u}(z) \\
& =\sum_{\alpha} \sum_{\beta} c_{i, \alpha} \overline{w_{i}^{\beta}} \hat{P}_{\beta} \circ \varphi_{u}(z) F_{\alpha} \circ \varphi_{u}(z) k_{u}(z) .
\end{aligned}
$$

and

$$
\begin{aligned}
\sum_{i} p_{i}(z) f_{i}(z) & =\sum_{\alpha} \sum_{\beta} \sum_{i} c_{i, \alpha} \overline{w_{i}^{\beta}} \hat{P}_{\beta} \circ \varphi_{u}(z) F_{\alpha} \circ \varphi_{u}(z) k_{u}(z) \\
& =\sum_{\alpha} \sum_{\beta} \delta_{\alpha, \beta} \hat{P}_{\beta} \circ \varphi_{u}(z) F_{\alpha} \circ \varphi_{u}(z) k_{u}(z) \\
& =\sum_{\alpha} \hat{P}_{\alpha} \circ \varphi_{u}(z) F_{\alpha} \circ \varphi_{u}(z) k_{u}(z) .
\end{aligned}
$$

From this it is easy to see that the functions $p_{i}$ and $f_{i}$ satisfy inequalities (i) and (ii).

For each $\zeta \in A \cap \partial \mathbb{B}_{n}$ we have found a neighborhood $U_{\zeta}:=U$ such that for any $u \in U_{\zeta}$, we have a decomposition with the stated properties. By compactness, we can cover $A \cap \partial \mathbb{B}_{n}$ by finitely many such neighborhoods. This proves (1).

Next we prove (2). Suppose $v \in \mathbb{B}_{n}$ and $\beta(v, A)>5 s^{\prime} \log 2$. By Lemma $5.1(2), F_{v}^{\prime}=E_{v 3}^{\prime} \subset$ $D\left(v, 4 s^{\prime} \log 2\right)$. Let $\xi \in A$ be a point such that $\beta(v, \xi)=\beta(v, A)$. Since

$$
\beta\left(\varphi_{\xi}(v), 0\right)=\beta(v, \xi)=\beta(v, A)>5 s^{\prime} \log 2,
$$

by Lemma $2.11(2)$,

$$
1-\left|\varphi_{\xi}(v)\right|^{2} \leq 4 e^{-2 \beta(\xi, v)}<2^{-10 s^{\prime}+2} .
$$

By [19, 2.2.7], the Euclidean diameter of $D\left(\varphi_{\xi}(v), 4 s^{\prime} \log 2\right)$ do not exceed $2^{-s^{\prime}}$. Since $\varphi_{\xi}\left(F_{v}^{\prime}\right) \subset$ $\varphi_{\xi}\left(D\left(v, 4 s^{\prime} \log 2\right)\right)=D\left(\varphi_{\xi}(v), 4 s^{\prime} \log 2\right)$, the Euclidean diameter of $\varphi_{\xi}\left(F_{v}^{\prime}\right)$ do not exceed $2^{-s^{\prime}}$.

Also, we can assume that $v$ is close enough to $\partial \mathbb{B}_{n}$ (equivalently, $k$ is large enough) so that $\xi$ is a regular point of $A$. A simple computation (cf. [21] Lemma 3.12) shows that $\varphi_{\xi}(v)$ is perpendicular to $T_{0} \varphi_{\xi}(A)=\varphi_{\xi}\left(T_{\xi} A+\xi\right)$ (cf. [19, Proposition 2.4.2]).

Let $\delta>0$ be a sufficiently small constant to be determined later. Suppose $\zeta \in A \cap \partial \mathbb{B}_{n}$. In the proof of (1), we have constructed an open neighborhood $U$ of $\zeta$ and an open subset $\mathcal{W}$ in $\mathbb{C}^{n-d}$ such that for any $u \in U \cap A$ and any $w \in \mathcal{W}$,

$$
\psi_{w}(z)=\frac{P_{\left.\pi_{u}\right|_{A}}(z, w)}{\hat{P}_{\left.\pi_{u}\right|_{A}}(z, w)}
$$

is non-vanishing on $U_{2}$, where $U_{2}$ is another open neighborhood of $\zeta$. Here the canonical defining functions are constructed based on the basis $\left\{e_{u, 1}, \cdots, e_{u, n}\right\}$ as in the proof of (1). For $v$ close enough to $\zeta$ we can assume that $u \in U$ and $F_{v}^{\prime} \subset U_{2}$. Thus by shrinking $U$ we will have that $\psi_{w}(z)$ is non-vanishing on $F_{v}^{\prime}$ for any $v \in U$.

Notice that although $\mathcal{W}$ depends on our choice of $\delta$, from the proof of Theorem 8.5 (which is used in the proof of (1)), by shrinking the set $U$, we can always ensure that the volume of $\Pi(\mathcal{W})$ is greater than half of the volume of $\mathbb{P}_{n-d-1}$. Here $\Pi$ denotes the canonical map from $\mathbb{C}^{n-d} \backslash\{0\}$ to $\mathbb{P}_{n-d-1}$. 
On the other hand, from the previous argument, we know that the Euclidean diameter of $\varphi_{\xi}\left(F_{v}^{\prime}\right)$ is less than $2^{-s^{\prime}}, \varphi_{\xi}(v)$ is perpendicular to $\varphi_{\xi}\left(T_{\xi} A+\xi\right)$, and that $1-\left|\varphi_{\xi}(v)\right|^{2} \leq 4 e^{-2 \beta(\xi, v)}<$ $2^{-10 s^{\prime}+2}$. By Lemmas 4.4, 4.5 and our construction in (1), for $v$ close enough to $\partial \mathbb{B}_{n}$, we will have

$$
\operatorname{dist}_{H}\left(\varphi_{\xi}(A) \cap D(0,5 s \log 2), L_{\xi} \cap D(0,5 s \log 2)\right) \leq 2 \delta .
$$

Denote $\mathcal{W}_{1}=\left\{w \in \mathbb{C}^{n-d}: \hat{P}_{\left.\pi_{\xi}\right|_{\varphi_{\xi}(A)}}(z, w)\right.$ is non-vanishing on $\left.\varphi_{\xi}\left(F_{v}^{\prime}\right)\right\}$. For $s^{\prime}$ large enough and $\delta$ small enough, we will have that the volume of $\Pi\left(\mathcal{W}_{1}\right)$ is greater than half of the volume of $\mathbb{P}_{n-d-1}$. Choose such a $\delta$. Then $\mathcal{W} \cap \mathcal{W}_{1}$ is non-empty. Choose a $w_{0} \in \mathcal{W} \cap \mathcal{W}_{1}$. Let $U$ be the open neighborhood of $\zeta$ determined by $\delta$. Then for any $v \in U$, by (6.11) and the fact that $w_{0} \in \mathcal{W}_{1}, \hat{P}_{\left.\pi_{\xi}\right|_{A}}\left(z, w_{0}\right)$ is non-vanishing on $F_{v}^{\prime}$. Since $w_{0} \in \mathcal{W}, \frac{P_{\left.\pi_{\xi}\right|_{A}}\left(z, w_{0}\right)}{\hat{P}_{\left.\pi_{\xi}\right|_{A}}\left(z, w_{0}\right)}$ is also non-vanishing on $F_{v}^{\prime}$. Thus $P_{\pi_{\xi} \mid A}\left(z, w_{0}\right)$ is non-vanishing on $F_{v}^{\prime}$. By Theorem 8.3 and Hilbert's Nullstellensatz, $P_{\left.\pi_{\xi}\right|_{A}}\left(z, w_{0}\right)$ is a polynomial of degree less than $N$ in $J$. Thus $p=P_{\left.\pi_{\xi}\right|_{A}}^{m}\left(z, w_{0}\right) \in J^{m}$ is nonvanishing on $F_{v}^{\prime}$ and $\operatorname{deg} p \leq N$. This proves (2).

We are ready to prove Lemma 6.1,

Proof of Lemma 6.1 . Notice that the varieties $Z_{j}$ are disjoint in a neighborhood of $\partial \mathbb{B}_{n}$. If $\zeta \in Z_{j} \cap \partial \mathbb{B}_{n}$, then for each $i \neq j$, there exists $p_{i} \in I_{i}^{m_{i}}$ such that $p_{i}(\zeta) \neq 0$. Thus for each $\zeta \in Z_{j} \cap \partial \mathbb{B}_{n}$, we can find a neighborhood $V_{\zeta}$ and $k-1$ polynomials $p_{i} \in I_{i}^{m_{i}}, i \neq j$ such that $p_{i}$ are non-vanishing on $V_{\zeta}$. Choose finitely many open sets $V_{\zeta}$ that cover $Z \cap \partial \mathbb{B}_{n}$. Suppose $F_{u} \subset V_{\zeta}$, where $\zeta \in Z_{j} \cap \partial \mathbb{B}_{n}$. For $f \in I$, let $P_{l}, f_{l}$ be the functions constructed in Lemma 6.5, for $I_{j}^{m_{j}}$. Then we can simply replace $P_{l}$ with $p_{l} \Pi_{i \neq j} p_{i}$ and $f_{l}$ with $\frac{f_{l}}{\Pi_{i \neq j} p_{j}}$. This proves $(1)$. The proof for (2) is similar.

We are ready to prove Theorem 4.1.

Proof of Theorem 4.1. For any $\epsilon>0$ sufficiently small, let $C$ be the constant in Lemma 6.1. Choose $R_{0}>0$ so that $C \epsilon_{R_{0}-3}^{2}<\epsilon$. Let $\left\{E_{u}\right\}_{u \in \mathcal{L}},\left\{E_{v}^{\prime}\right\}_{v \in \mathcal{L}^{\prime}},\left\{F_{u}\right\}_{u \in \mathcal{L}},\left\{F_{v}^{\prime}\right\}_{v \in \mathcal{L}^{\prime}}$ be determined by $R$ as in Section 5. Let $K, N$ be the positive integers in Lemma 6.1. Let $\delta=\sqrt{1-2^{-2 m(K+1)}}$. Finally, let $\left\{E_{i}\right\}=\left\{E_{u}\right\}_{u \in \cup_{k} \geq K} \mathcal{L}_{k} \cup\left\{E_{v}^{\prime}\right\}_{v \in \cup_{k \geq 10 K} \mathcal{L}_{k}^{\prime}}$. The polynomials $p_{i j}$ will be the corresponding polynomials in Lemma 6.1. The conditions (1) and (2) in Hypothesis 1 follow from Lemma 5.1, where we take $M=2^{10 n+3}$. The conditions (3) and (4)(i)(ii)(iv) follow from Lemma 6.1. By Lemma 5.1 (2), $\left(1-|\lambda|^{2}\right)$ is comparable to $\left(1-|u|^{2}\right)$ for $\lambda \in F_{u}$ and $\left(1-|\lambda|^{2}\right)$ is comparable to $\left(1-|v|^{2}\right)$ for $\lambda \in F_{v}^{\prime}$. From this, condition (4)(iii) follows immediately.

Therefore I satisfies Hypothesis 1. The rest of the theorem follow from Theorem 3.4. This completes the proof.

\section{Concluding Remarks}

In this paper, we have provided a unified proof for most known results of the ArvesonDouglas Conjecture. In fact, we have proved the stronger result that the submodules under our consideration have the asymptotic stable division property. We raise the following question.

Question: Suppose $I$ is an ideal in $\mathbb{C}\left[z_{1}, \cdots, z_{n}\right]$. Find sufficient conditions for $I$ to have the asymptotic stable division property. Find sufficient conditions for $I$ to have the asymptotic 
stable division property with generating elements $\left\{h_{i}\right\}$ being polynomials of uniformly bounded degrees.

By Theorem 3.2, a positive result on this question will lead to a positive result of the ArvesonDouglas Conjecture. We would also like to explore other applications of the asymptotic stable division property, for example, in index theory.

The techniques we have developed in this paper are aimed at getting more general results. For the next step, we plan to consider the following examples.

(1) Arbitrary union of smooth, transversal varieties.

(2) Varieties with certain type of singular points on $\partial \mathbb{B}_{n}$. For example, singular points $\zeta$ with tangent cones being linear subspaces. This will cover the classic example of singular point, $z_{1}^{2}=z_{2}^{3}$ at 0 .

Tools, for example, from [6] and [18] can be useful.

We will also use the techniques to study the Arveson-Douglas Conjecture in connection with the $L^{2}$-extension problem [25]. The covering constructed in Section 5 can be useful in constructing a holomorphic extension.

\section{Appendix}

For an algebraic set $A \subset \mathbb{C}^{n}$ with pure dimension, we can show that the functions $P_{\pi}$ and $P_{\pi, \alpha}$ in Definition 2.6 are polynomials in $z$ and $\bar{w}:=\left(\bar{w}_{p+1}, \cdots, \bar{w}_{n}\right)$. Let us first consider a simple case.

Lemma 8.1. Suppose $A \subset \mathbb{C}^{n}$ is an algebraic set of pure dimension $n-1$, and suppose

$$
\pi: A \rightarrow \mathbb{C}^{n-1}, z \mapsto z^{\prime}:=\left(z_{1}, \cdots, z_{n-1}\right)
$$

is proper. Then $P_{\pi}(z, w)$ and $P_{\pi, \alpha}(z)$ are polynomials in $z$ and $\bar{w}$.

Proof. In this case, there is only one canonical defining function. If $\pi$ is $k$-sheeted, then $\alpha=k$, and

$$
P_{\pi}\left(z, w_{n}\right)=P_{\pi, k}(z) \bar{w}_{n}^{k}
$$

It suffices to show that $P_{\pi, k}$ is a polynomial. By definition, $P_{\pi, k}$ is the Weierstrass polynomial determined by $\pi$.

The algebraic set $A$ decomposes into finitely many irreducible algebraic sets. It is easy to see that $P_{\pi, k}$ is just the product of the canonical defining functions of the irreducible components. Without loss of generality, we can assume $A$ is itself irreducible.

By [16, Proposition 1.13], there is an irreducible polynomial $Q(z)$ such that $A=Z(Q)$. Clearly $\operatorname{deg}_{z_{n}} Q \geq k$. Write $l=\operatorname{deg}_{z_{n}} Q$ and write $Q$ as a polynomial in $z_{n}$ with coefficients in $\mathbb{C}\left[z_{1}, \cdots, z_{n-1}\right]$

$$
Q(z)=q_{l}\left(z^{\prime}\right) z_{n}^{l}+\cdots
$$

Consider the set

$$
B=\left\{z \in A: q_{l}\left(z^{\prime}\right)=0, \text { or } \partial_{n} Q(z)=0\right\} .
$$

$\pi(B)$ is the set of points $z^{\prime} \in \mathbb{C}^{n-1}$ such that $Q\left(z^{\prime}, \cdot\right)$ do not have $l$ distinct simple roots. Since $Q$ is irreducible, $B$ is an analytic subset of dimension $\leq n-2$. By [6. Proposition 3.3.2], $\pi(B) \subset \mathbb{C}^{n-1}$ is an analytic subset of dimension $\leq n-2$. Thus $\mathbb{C}^{n-p} \backslash \pi(B)$ is dense in $\mathbb{C}^{n-1}$. 
If $l>k$, for any $z^{\prime} \in \mathbb{C}^{n-1} \backslash \pi(B), \pi^{-1}\left(z^{\prime}\right)$ contains $l>k$ distinct points, a contradiction. Thus $\operatorname{deg}_{z_{n}} Q=k$. Also, if $\operatorname{deg} q_{k}>0$, then for $z^{\prime} \notin \sigma$, comparing the two polynomials in $z_{n}$,

$$
Q\left(z^{\prime}, z_{n}\right)=q_{k}\left(z^{\prime}\right) z_{n}^{k}+\cdots
$$

and

$$
P_{\pi, k}\left(z^{\prime}, z_{n}\right)=\left(z_{n}-a_{1}\left(z^{\prime}\right)\right) \cdots\left(z_{n}-a_{k}\left(z^{\prime}\right)\right)
$$

we get $Q\left(z^{\prime}, z_{n}\right)=q_{k}\left(z^{\prime}\right) P_{\pi, k}\left(z^{\prime}, z_{n}\right), z^{\prime} \notin \sigma, z_{n} \in \mathbb{C}$. Since $\sigma$ is nowhere dense, $Q \equiv q_{k} P_{\pi, k}$. But then if $q_{k}\left(z^{\prime}\right)=0$ at some $z^{\prime}$, we will have infinitely many points on the fiber $\pi^{-1}\left(z^{\prime}\right)$, a contradiction. Thus $q_{k}$ is a constant. Without loss of generality, we assume $q_{k} \equiv 1$. Then $P_{\pi, k}=Q$ is a polynomial. This completes the proof.

Lemma 8.2. For any open set $U \subset \mathbb{B}_{n}$ and any finite collection of indexes

$$
F \subset\left\{\alpha=\left(\alpha_{1}, \cdots, \alpha_{n}\right): \alpha_{i} \in \mathbb{N}\right\},
$$

let $K=\# F$. Then there exists $K$ distinct points $\left\{w_{i}\right\}_{i=1}^{k}$ in $U$ such that the vectors $\left\{\left(w_{i}^{\alpha}\right)_{\alpha \in F}\right\}_{i=1}^{K}$ in $\mathbb{C}^{K}$ are linearly independent.

Proof. Let

$$
L=\operatorname{span}\left\{\left(w^{\alpha}\right)_{\alpha \in F}: w \in U\right\} .
$$

It suffices to show that $L=\mathbb{C}^{K}$. Otherwise, choose a non-zero vector $\left(a_{\alpha}\right)_{\alpha \in F}$ that is perpendicular with $L$. Define

$$
f(w)=\sum_{\alpha \in F} \overline{a_{\alpha}} w^{\alpha}
$$

Then $f$ is a analytic polynomial that vanishes on the open set $U$. Therefore $f$ is identically zero. So $a_{\alpha}=0, \forall \alpha \in F$, a contradiction. This completes the proof.

Theorem 8.3. Suppose $A \subset \mathbb{C}^{n}$ is an algebraic set of pure dimension $p$. Suppose the projection

$$
\pi: A \rightarrow \mathbb{C}^{p}, z \mapsto z^{\prime}:=\left(z_{1}, \cdots, z_{p}\right)
$$

is proper. Then the functions $P_{\pi}(z, w)$ and $P_{\pi, \alpha}(z)$ are polynomials in $z$ and $\bar{w}$. Moreover, there exists a positive integer $N$, depending only on $A$, such that for any choice of basis and any proper projection $\pi$, the degrees of $P_{\pi}(z, w)$ (in $\left.z\right)$ and $P_{\pi, \alpha}$ are less than $N$.

Proof. Let $\sigma \subset \mathbb{C}^{p}$ be the critical set of $\pi$. Suppose $\pi$ is $k$-sheeted. Fix any $z^{\prime} \notin \sigma$, we have $\pi^{-1}\left(z^{\prime}\right)=\left\{\left(z^{\prime}, a_{1}\left(z^{\prime}\right)\right), \cdots,\left(z^{\prime}, a_{k}\left(z^{\prime}\right)\right)\right\}$, where $\left\{a_{1}\left(z^{\prime}\right), \cdots, a_{k}\left(z^{\prime}\right)\right\}$ are $k$ distinct points in $\mathbb{C}^{n-p}$. The set

$$
\mathcal{W}:=\left\{w \in \mathbb{C}^{n-p}:\left\langle a_{1}\left(z^{\prime}\right), w\right\rangle, \cdots,\left\langle a_{k}\left(z^{\prime}\right), w\right\rangle \text { are distinct at least for one } z^{\prime} \in U^{\prime}\right\}
$$

is open in $\mathbb{C}^{n-p}$. Fix any $w \in \mathcal{W}$. Consider the projections

$$
\pi_{w}: A \rightarrow \mathbb{C}^{p+1}, z \mapsto\left(z^{\prime},\left\langle z^{\prime \prime}, w\right\rangle\right)
$$

and

$$
\pi_{w}^{\prime}: \pi_{w}(A) \subset \mathbb{C}^{p+1} \rightarrow \mathbb{C}^{p},\left(z^{\prime},\left\langle z^{\prime \prime}, w\right\rangle\right) \rightarrow z^{\prime} .
$$

Then $\pi=\pi_{w}^{\prime} \circ \pi_{w}$. By [6, $3.1(2)$ ], both $\pi_{w}$ and $\pi_{w}^{\prime}$ are proper maps. By [6, Theorem 3.2, Proposition 3.3.2], $\pi_{w}(A)$ is a pure algebraic set in $\mathbb{C}^{p+1}$ of dimension $p$, and $\pi_{w}^{\prime}$ is a $k$-sheeted analytic cover. By Lemma 8.1, $P_{\pi_{w}^{\prime}}\left(\left(z^{\prime}, \lambda\right), \xi\right)$ is a polynomial in $z^{\prime}, \lambda$ and $\bar{\xi}$. Also, from the proofs of [6. Theorem 3.2, Proposition 3.3.2], the degree (in $\left.\left(z^{\prime}, \lambda\right)\right)$ of $P_{\pi_{w}^{\prime}}\left(\left(z^{\prime}, \lambda\right)\right.$, $\xi$ ) has a upper 
bound determined by any set of generators of the ideal $\left\{p \in \mathbb{C}\left[z_{1}, \cdots, z_{n}\right]:\left.p\right|_{A}=0\right\}$, which we denote by $N$. Checking by definition, we have the equation

$$
P_{\pi}(z, w)=P_{\pi_{w}^{\prime}}\left(\left(z^{\prime},\left\langle z^{\prime \prime}, w\right\rangle\right), 1\right) .
$$

Thus for any $w \in \mathcal{W}, P_{\pi}(z, w)$ is a polynomial in $z$ and $\operatorname{deg}_{z} P_{\pi}(z, w) \leq N$. Fix an order of the set $\Gamma=\left\{\alpha=\left(\alpha_{p+1}, \cdots, \alpha_{n}\right):|\alpha|=k\right\}$, and let $K=\# \Gamma$. By Lemma 8.2 , we can choose $K$ distinct points $\left\{w_{j}\right\}_{j=1}^{K} \subset \mathcal{W}$ such that the $K \times K$ matrix $W=\left(\overline{w_{j}^{\alpha}}\right)_{\alpha \in \Gamma, j=1, \cdots, K}$ is invertible. From the equations

$$
P_{\pi}\left(z, w_{j}\right)=\sum_{\alpha \in \Gamma} P_{\pi, \alpha}(z) \overline{w_{j}^{\alpha}}, \quad j=1, \cdots, K,
$$

we can solve $P_{\pi, \alpha}$ as linear combinations of $\left\{P_{\pi}\left(z, w_{j}\right)\right\}_{j=1}^{K}$. Thus $P_{\pi, \alpha}$ are polynomials in $z$, and then $P_{\pi}(z, w)$ is a polynomial in $z$ and $\bar{w}$. Moreover, $\operatorname{deg}_{z} P_{\pi}(z, w) \leq N, \operatorname{deg} P_{\pi, \alpha}(z) \leq N$. This completes the proof.

Let $\mathbb{P}_{n}$ denote the $n$-dimensional complex projective space. Then $\mathbb{C}^{n}$ can be viewed as a subset of $\mathbb{P}_{n}$ via the natural embedding

$$
\mathbb{C}^{n} \rightarrow \mathbb{P}_{n}, \quad\left(z_{1}, \cdots, z_{n}\right) \mapsto\left[1, z_{1}, \cdots, z_{n}\right] .
$$

For any algebraic set $A \subset \mathbb{C}^{n}$, its closure $\bar{A}$ in $\mathbb{P}_{n}$ is an analytic subset of $\mathbb{P}_{n}$. For a $p$-dimensional linear space $L \subset \mathbb{C}^{n}, \bar{L}$ is a $p$-dimensional linear space in $\mathbb{P}_{n}$. Its orthogonal complement in $\mathbb{P}_{n}$ is of dimension $n-p-1$.

$$
\bar{L}^{\perp}=\left\{\left[z_{0}, \cdots, z_{n}\right]:\left(z_{0}, \cdots, z_{n}\right) \perp(1, w), w \in L\right\} .
$$

We have the following lemma [6, 7.3].

Lemma 8.4. Let $A$ be a pure p-dimensional projective algebraic set in $\mathbb{P}_{n}$ and let $L \subset \mathbb{P}_{n}$ be a complex $n-p-1$-dimensional plane not intersecting $A$. Then the projection $\pi: A \rightarrow L^{\perp}$ is proper.

The following theorem will be used in the proof of Theorem 4.1.

Theorem 8.5. Suppose $A \subset \mathbb{C}^{n}$ is an algebraic set of pure dimension $p<n$ and $0 \in A$. Assume the following.

(1) Denote $L=\left\{\left(z^{\prime}, 0\right): z^{\prime} \in \mathbb{C}^{p}\right\} \subset \mathbb{C}^{n}$ and suppose $\bar{L}^{\perp} \cap \bar{A}=\emptyset$. Therefore if we denote $\pi: \mathbb{C}^{n} \rightarrow \mathbb{C}^{p}, z \mapsto z^{\prime}:=\left(z_{1}, \cdots, z_{p}\right)$, then $\left.\pi\right|_{A}$ is proper.

(2) $U=U^{\prime} \times U^{\prime \prime}$, where $0 \in U^{\prime} \subset \mathbb{C}^{p}$ and $0 \in U^{\prime \prime} \subset \mathbb{C}^{n-p}$ are open sets. $\left.\pi\right|_{A \cap U}$ is also proper. Moreover, $\pi^{-1}(0) \cap A \cap U=\{0\}$.

Then there exist open sets $0 \in U_{1}^{\prime} \subset U^{\prime}, 0 \in U_{1}^{\prime \prime} \subset U^{\prime \prime}$, an open neighborhood $\mathcal{U}$ of the $n \times n$ identity matrix $I_{n \times n}$ in $U(n)$, and an open set $\mathcal{W} \subset \mathbb{C}^{n-p}$ with the following properties. Denote $U_{1}=U_{1}^{\prime} \times U_{1}^{\prime \prime}$. For any $l \in \mathcal{U}$, the projections $\left.\pi\right|_{l(A)}$ and $\left.\pi\right|_{l(A) \cap U_{1}}$ are proper. Moreover, for any $w \in \mathcal{W}$, the function

$$
\psi_{w}(z)=\frac{P_{\left.\pi\right|_{l(A)}}(z, w)}{P_{\left.\pi\right|_{l(A) \cap U_{1}}}(z, w)}
$$

is non-vanishing and holomorphic in $U_{1}$.

Proof. Since $\left.\pi\right|_{A}$ is proper, we know that $\pi^{-1}(0) \cap A$ consists of finitely many points. Suppose

$$
\left(\pi^{-1}(0) \cap A\right) \backslash\{(0,0)\}=\left\{\left(0, a_{1}\right), \cdots,\left(0, a_{k}\right)\right\},
$$


where $a_{i} \in \mathbb{C}^{n-p}$. Take an open set $\mathcal{W} \subset \mathbb{C}^{n-p}$ whose closure is contained in the open set $\left\{w \in \mathbb{C}^{n-p}:\left\langle a_{i}, w\right\rangle \neq 0\right\}$. We can find open neighborhoods $a_{i} \in V_{i} \subset \mathbb{C}^{n-p}, 0 \in U_{1}^{\prime \prime} \subset \mathbb{C}^{n-p}$ such that

$$
\left\langle\lambda-z^{\prime \prime}, w\right\rangle \neq 0, \quad \forall i, \forall \lambda \in V_{i}, \forall z^{\prime \prime} \in U_{1}^{\prime \prime}, \forall w \in \mathcal{W} .
$$

We claim that there exist an open neighborhood $\mathcal{U}$ of $I_{n \times n}$ in $U(n)$ and an open set $0 \in U_{1}^{\prime} \subset U^{\prime}$ with the following property. Denote $U_{1}=U_{1}^{\prime} \times U_{1}^{\prime \prime}$. Then $\left.\pi\right|_{l(A)}$ and $\left.\pi\right|_{l(A) \cap U_{1}}$ are proper. Moreover,

$$
\pi^{-1}\left(U_{1}^{\prime}\right) \cap l(A) \subset U_{1} \cup\left(\bigcup_{i=1}^{k} U_{1}^{\prime} \times V_{i}\right) .
$$

By assumption, $\bar{L}^{\perp} \cap \bar{A}=\emptyset$. Therefore we can find an open neighborhood $\mathcal{U}$ of $I_{n \times n}$ in $U(n)$ such that $\forall l \in \mathcal{U}, \overline{l^{-1}(L)} \perp \cap \bar{A}=\emptyset$. Thus the projection from $A$ onto $l^{-1}(L)$ is proper. Equivalently, $\left.\pi\right|_{l(A)}$ is proper. Also, by the proof of [6, Theorem 7.4.2], $A$ is contained in the union of a ball $B:=\{z:|z|<R\}$ and a cone $K:=\left\{z:\left|z^{\prime \prime}\right|<C\left|z^{\prime}\right|\right\}$.

On the other hand, by [6, Corollary 4.2], we can take $U_{2}^{\prime}$ small enough so that

$$
\pi^{-1}\left(U_{2}^{\prime}\right) \cap A \subset\left(U_{2}^{\prime} \times U_{1}^{\prime \prime}\right) \cup\left(\bigcup_{i=1}^{k} U_{2}^{\prime} \times V_{i}\right) .
$$

If we shrink $\mathcal{U}$, we can ensure that $\forall l \in \mathcal{U}, l^{-1} \pi^{-1}\left(U_{2}^{\prime}\right) \backslash B$ is outside the cone $K$. Then $l^{-1} \pi^{-1}\left(U_{2}^{\prime}\right) \cap A \subset B$. Then if we shrink $\mathcal{U}$ again, we can find $0 \in U_{1}^{\prime} \subset U_{2}^{\prime}$ so that $\forall l \in \mathcal{U}$, $l^{-1} \pi^{-1}\left(U_{1}^{\prime}\right) \cap B \subset \pi^{-1}\left(U_{2}^{\prime}\right)$. Thus

$$
l^{-1} \pi^{-1}\left(U_{1}^{\prime}\right) \cap A=l^{-1} \pi^{-1}\left(U_{1}^{\prime}\right) \cap B \cap A \subset \pi^{-1}\left(U_{2}^{\prime}\right) \cap A \subset\left(U_{2}^{\prime} \times U_{1}^{\prime \prime}\right) \cup\left(\bigcup_{i=1}^{k} U_{2}^{\prime} \times V_{i}\right) .
$$

If we replace the right hand side with a compact neighborhood $\mathcal{N}$ of $A \cap \pi^{-1}\left(U_{1}^{\prime}\right)$ contained in $\left(U_{2}^{\prime} \times U_{1}^{\prime \prime}\right) \cup\left(\bigcup_{i=1}^{k} U_{2}^{\prime} \times V_{i}\right)$, then the same method will give us $l^{-1} \pi^{-1}\left(U_{1}^{\prime}\right) \cap A \subset \mathcal{N}$. Then we can shrink $\mathcal{U}$ again to ensure $l(\mathcal{N}) \subset\left(U_{2}^{\prime} \times U_{1}^{\prime \prime}\right) \cup\left(\bigcup_{i=1}^{k} U_{2}^{\prime} \times V_{i}\right)$. Then we have $\forall l \in \mathcal{U}$,

$$
\pi^{-1}\left(U_{1}^{\prime}\right) \cap l(A) \subset\left(U_{2}^{\prime} \times U_{1}^{\prime \prime}\right) \cup\left(\bigcup_{i=1}^{k} U_{2}^{\prime} \times V_{i}\right)
$$

Then obviously,

$$
\pi^{-1}\left(U_{1}^{\prime}\right) \cap l(A) \subset\left(U_{1}^{\prime} \times U_{1}^{\prime \prime}\right) \cup\left(\bigcup_{i=1}^{k} U_{1}^{\prime} \times V_{i}\right)
$$

This proves our claim.

The open sets $U_{1}^{\prime \prime}$ and $V_{i}$ can be chosen to be disjoint. By [6, $\left.3.1(3)\right],\left.\pi\right|_{l(A) \cap U_{1}}$ is also proper. Let $z^{\prime} \in U_{1}^{\prime}$ be outside the critical sets of both projections. Then

$$
\pi^{-1}\left(z^{\prime}\right) \cap l(A)=\left\{\left(z^{\prime}, b_{1}\left(z^{\prime}\right)\right), \cdots,\left(z^{\prime}, b_{l}\left(z^{\prime}\right)\right) ;\left(z^{\prime}, a_{1}\left(z^{\prime}\right)\right), \cdots,\left(z^{\prime}, a_{k}\left(z^{\prime}\right)\right)\right\} .
$$

Here $b_{j}\left(z^{\prime}\right) \in U_{1}^{\prime \prime}, a_{j}\left(z^{\prime}\right) \in \cup_{i} V_{i}$. By definition, for $z \in U_{1}$ and $w \in \mathcal{W}$,

$$
P_{\left.\pi\right|_{l(A)}}(z, w)=\prod_{j=1}^{l}\left\langle z^{\prime \prime}-b_{j}\left(z^{\prime}\right), w\right\rangle \times \prod_{j=1}^{k}\left\langle z^{\prime \prime}-a_{j}\left(z^{\prime}\right), w\right\rangle=P_{\left.\pi\right|_{l(A) \cap U_{1}}}(z, w) \times \psi_{w}(z),
$$


where

$$
\psi_{w}(z)=\Pi_{j=1}^{k}\left\langle z^{\prime \prime}-a_{j}\left(z^{\prime}\right), w\right\rangle .
$$

From our construction, it is straightforward that $\psi_{w}$ is non-vanishing on $U_{1}$. This completes the proof.

Theorem 8.6. Suppose $A$ is a p-dimensional irreducible affine algebraic set in $\mathbb{C}^{n}$ and $0 \in A$. Assume that $A \nsubseteq e_{n}^{\perp}$. Let

$$
\mathcal{G}_{n}=\left\{L \in G(p, n): e_{n} \in L\right\}
$$

and

$$
\tilde{\mathcal{G}}_{n}=\left\{L \in \mathcal{G}_{n}: \bar{L}^{\perp} \cap \bar{A}=\emptyset\right\} .
$$

Here $G(p, n)$ is the Grassmannian. Then $\tilde{\mathcal{G}}_{n}$ is a dense open set in $\mathcal{G}_{n}$. Equivalently, let

$$
\tilde{\mathcal{U}}_{n}=\left\{l \in U(n-1): \operatorname{span}\left\{l\left(e_{1}\right), \cdots, l\left(e_{p-1}\right), e_{n}\right\} \in \tilde{\mathcal{G}}_{n}\right\} .
$$

Then $\tilde{\mathcal{U}}_{n}$ is dense in $U(n-1)$.

Proof. The two statements are clearly equivalent. Let us prove the first statement. Consider the canonical projection.

$$
\Pi: \mathbb{C}^{n+1} \backslash\{0\} \rightarrow \mathbb{P}_{n}, \quad\left(z_{0}, z_{1}, \cdots, z_{n}\right) \mapsto\left[z_{0}, z_{1}, \cdots, z_{n}\right] .
$$

For $L \in G(p, n)$, denote $\tilde{L}=\Pi^{-1}\left(\bar{L}^{\perp}\right) \cup\{0\}$ and $\tilde{A}=\Pi^{-1}(\bar{A}) \cup\{0\}$. Then $\bar{L}^{\perp} \cap \bar{A}=\emptyset$ if and only if $\tilde{L} \cap \tilde{A}=\{0\}$. Since $A$ is irreducible and has dimension $p, \tilde{A}$ is a homogeneous irreducible algebraic set of dimension $p+1$. $\tilde{L}$ is a linear subspace of dimension $n-p$. The condition that $e_{n} \in L$ is equivalent to that $\tilde{L} \subset L_{n}:=e_{n}^{\perp} \subset \mathbb{C}^{n+1}$. Let $\tilde{A}_{n}:=\tilde{A} \cap L_{n}$. Then $L \in \tilde{\mathcal{G}}_{n}$ if and only if $\tilde{L} \subset L_{n}$ and $\tilde{L} \cap \tilde{A}_{n}=\{0\}$.

We claim that $\operatorname{dim} \tilde{A}_{n} \leq p$. Otherwise, $\operatorname{dim} \tilde{A}_{n}=p+1$. Since $\tilde{A}$ is irreducible, it cannot properly contain any algebraic set of the same dimension. So $\tilde{A}=\tilde{A}_{n}$ and therefore $\tilde{A} \subset L_{n}$. However, this implies that $A=\bar{A} \cap \mathbb{C}^{n} \subset \Pi(\tilde{A} \backslash\{0\}) \cap \mathbb{C}^{n} \subset \Pi\left(L_{n} \backslash\{0\}\right) \cap \mathbb{C}^{n}=e_{n}^{\perp}$. A contradiction. Thus $\operatorname{dim} \tilde{A} \leq p$.

Assume $\tilde{L} \subset L_{n}$. Both $\tilde{L}$ and $\tilde{A}_{n}$ are homogeneous varieties in $L_{n} \cong \mathbb{C}^{n}$. Thus $\tilde{L} \cap \tilde{A}_{n}=\{0\}$ if and only if their preimages in $\mathbb{P}_{n-1}$ do not intersect. The preimages of the two varieties have dimension $n-p-1, \leq p-1$, respectively. Thus the set of $\tilde{L} \subset L_{n}$ not intersecting $\tilde{A}$ form a dense open sent in $G(n-p, n)$. From this it is easy to see that $\tilde{\mathcal{G}}_{n}$ is a dense open set in $\mathcal{G}_{n}$. This completes our proof.

\section{REFERENCES}

[1] William Arveson, Subalgebras of $C^{*}$-algebras. III. Multivariable operator theory. Acta Math. 181(1998), no. 2, 159-228.

[2] William Arveson, The curvature invariant of a Hilbert module over $\mathbb{C}\left[z_{1}, \cdots, z_{d}\right]$. J. Reine Angew. Math 522(2000), 173-236.

[3] William Arveson, The Dirac operator of a commuting $d$-tuple. J. Funct. Anal. 189(2002), no. 1, 53-79.

[4] William Arveson, $p$-summable commutators in dimension $d$. J. Operator Theory 54 (2005), no. 1, 101-117.

[5] William Arveson, Quotients of standard Hilbert modules. Trans. Amer. Math. Soc. 359 (2007), no. 12, 6027-6055.

[6] E. M. Chirka, Complex analytic sets. Mathematics and its Applications (Soviet Series), 46. Kluwer Academic Publishers Group, Dordrecht, 1989.

[7] Ronald G. Douglas, A new kind of index theorem. Analysis, geometry and topology of elliptic operators, 369-382, World Sci. Publ., Hackensack, NJ, 2006. 
[8] Ronald G. Douglas and Kai Wang, A harmonic analysis approach to essential normality of principal submodules. J. Funct. Anal. 261 (2001), no. 11, 3155-3180.

[9] Ronald G. Douglas, Kunyu Guo and Yi Wang, On the p-essential normality of principal submodules of the Bergman module on strongly pseudoconvex domains. submitted to Adv. Math.

[10] Ronald G. Douglas, Xiang Tang and Guoliang Yu, An analytic Grothendieck Riemann Roch theorem. Adv. Math. 294 (2016), no. 1, 3-19.

[11] Ronald G. Douglas and Yi Wang, Geometric Arveson-Douglas Conjecture and holomorphic extensions. Indiana Univ. Math. J. 66 (2017), no. 5, 1499-1535.

[12] Miroslav Engliš and Jörg Eschmeier, Geometric Arveson-Douglas conjecture. Adv. Math. 274 (2015), 606630 .

[13] Quanlei Fang and Jingbo Xia, Essential normality of polynomial-generated submodules: Hardy space and beyond, J. Funct. Anal. 265 (2013), 2991-3008.

[14] Quanlei Fang and Jingbo Xia, On the essential normality of principal submodules of the Drury-Arveson module, Indiana Univ. Math. J. 67 (2018), 1439-1498.

[15] Kunyu Guo and Kai Wang, Essentially normal Hilbert modules and K-homology. Math. Ann. 340 (2008), no. 4, 907-934.

[16] Robin Hartshorne, Algebraic geometry. Graduate Texts in Mathematics, No. 52. Springer-Verlag, New York-Heidelberg, 1977. xvi+496 pp. ISBN: 0-387-9024-9

[17] Matthew Kennedy and Orr Shalit, Essential normality and the decomposability of algebraic varieties. New York J. Math. 18 (2012), 877-890.

[18] José L. López and Nico M. Temme, Multi-point Taylor expansions of analytic functions Trans. Amer. Math. Soc. 356 (2004), no. 11, 4323-4342.

[19] W. Rudin, Function Theory on the Unit Ball of $\mathbb{C}^{n}$. Reprint of the 1980 edition. Classics in Mathematics. Springer-Verlag, Berlin, 2008. xiv+436 pp. ISBN: 978-3-540-68272-1.

[20] Orr Shalit, Stable polynomial division and essential normality of graded Hilbert modules. J. Lond. Math. Soc. (2) 83 (2011), no. 2, 273-289.

[21] Yi Wang and Ronald G. Douglas, Geometric Arveson-Douglas Conjecture - decomposition of varieties. Complex Anal. Oper. Theory 12 (2018), no. 5, 1267-1290.

[22] Yi Wang and Jingbo Xia, Essential normality for quotient modules and complex dimensions, J. Funct. Anal. (2018) https://doi.org/10.1016/j.jfa.2018.08.022

[23] Yi Wang and Jingbo Xia, Essential normality of principal submodules of the Hardy module on a pseudoconvex domain. submitted to J. Operator Theory.

[24] Jingbo Xia, A double commutant relation in the Calkin algebra on the Bergman space. J. Funct. Anal. 274 (2018), 1631-1656.

[25] Xiangyu Zhou, A survey on $L^{2}$ extension problem. Complex geometry and dynamics, 291-309, Abel Symp., 10, Springer, Cham, 2015.

Department of Mathematics, State University of New York at Buffalo, Buffalo, Ny 14260, USA

E-mail address: yiwangfdu@gmail.com 